\title{
VARIANZ IM REGELWERK \\ Bestattungsabläufe im Monumentalgrab von Anch-Hor, Obersthofmeister der Gottesgemahlin Nitokris (TT 414)
}

Von Julia Budka

\section{EinLeitung}

Welche Handlungssphären lassen sich aus dem fragmentarischen, materiellen Befund mehrfach benutzter altägyptischer Grabanlagen rekonstruieren? Dieser Frage soll beispielhaft im Rahmen des Anch-Hor-Projekts nachgegangen werden, und zwar anhand der Bearbeitung des Fundmaterials aus TT $414 \mathrm{im}$ Asasif, das eine zeitlichen Spanne vom 6. Jahrhundert v. Chr. bis ins 4. Jahrhundert $\mathrm{n}$. Chr. umfasst. ${ }^{1}$ Theoretische Grundlagen, methodische Ansätze und erste Ergebnisse werden in diesem Beitrag vorgestellt.

\subsection{Forschungsgeschichtliches zu Bestattungsab- läufen in ägyptischen Gräbern}

In jüngster Zeit wurde die deutliche Fokussierung ägyptischer Archäologie auf elitäre und königliche Gräber und Bestattungen wiederholt als Manko ausgewiesen, ja sogar als „tomb problem“ des Faches bezeichnet. ${ }^{2}$ Innerhalb der Betrachtungsweisen von funerären Relikten als wichtige Quel-

1 Das Projekt ist durch eine Kooperation der Humboldt Universität zu Berlin mit dem seinerzeitigen Konzessionsinhaber, Manfred Bietak, zustande gekommen. Ermöglicht wurden die bisherigen Arbeiten (2007-2009) durch die Gerda Henkel Stiftung, die Humboldt-Universität und das Österreichische Archäologische Institut Kairo. Teilnehmer der Kampagne 2009, auf deren Basis der vorliegende Beitrag erstellt wurde, waren: Julia Budka (Ägyptologin, Leitung vor Ort, Humboldt-Universität zu Berlin); Virgina Lynn Cashman (Ägyptologin, Oxford); Veronika Hinterhuber (Ägyptologin, Humboldt-Universität zu Berlin); Arvi Korhonen (Student, Humboldt-Universität zu Berlin); Karena Kuntze (Zeichnerin, Berlin); Erico Peintner und Ahmed Refaat Eisa Abo El-Ata (Restauratoren, Kairo); Hassan Ramadan Mahmoud (Ägyptologe, SCA Luxor); Nicole Richter (Studentin, Freie Universität Berlin) und Anja Wutte (Studentin, Universität Wien). Als Inspektor des ägyptischen Antikendienstes unterstützte uns Yassier Yussef Ahmed. Für die Arbeitserlaubnis sind wir wie immer Dr. Zahi Hawass und dem Permanent Committee des SCA zu Dank verpflichtet. Ohne die aktive Unterstützung und Zusammenarbeit mit den lokalen Behörden in Luxor wäre das Projekt nicht durchführbar gewesen. Wir danken besonders lengattung der altägyptischen Kultur hat es allerdings in den letzten Jahrzehnten zahlreiche neue, unterschiedliche Ansätze gegeben. Die als spezifisch altägyptisch geltenden, hohen materiellen Aufwendungen für das Leben nach dem Tod und die essentielle Sorge des Einzelnen, ein eigenes Grab zu Lebzeiten zu errichten und darin durch die Erinnerung und Kulthandlungen der Hinterbliebenen fortzudauern, ${ }^{3}$ werden zunehmend differenzierter betrachtet. Die aktuelle Forschung neigt dazu, diese Aspekte als generalisierendes Idealbild aufzufassen, das elitäre Privilegien aufzeigt und auch auf sozialen Ungleichheiten besteht. ${ }^{4}$ Vermehrt wird das dringende Desiderat angesprochen, das die Bearbeitung nicht-elitärer Bestattungen und Studie von Friedhöfen im ländlichen Milieu für das Fach darstellen. ${ }^{5}$

Da die Grablegung immer, selbst bei umfangreicher Vorsorge seitens des Verstorbenen zu Lebzeiten, durch die Hinterbliebenen erfolgt ${ }^{6}$ sind wohl auch im pharaonischen Ägypten Besonder-

Mansour Bourraik, Generaldirektor des Südlichen Oberägyptens; Mustafa Wasiri, Generaldirektor der Westseite Thebens; Nour Abdel Ghaffar, Direktor der Westseite; Abdel Ali Mohammed, Chefinspektor für Magazine und Museen sowie Fathy Yasin Abdel Kareem, Direktor der Mittleren Zone der Westseite; Ramadan Ahmed Ali, Chefinspektor der Mittleren Zone der Westseite; Abdel Nasser Mohammed Ahmed, Chefinspektor der Südlichen Zone der Westseite und Eez Eldin Kamal el-Nubi, Chefinspektor der Zone der Beamtengräber („Nobles Area“).

2 Richards 2005, 49-52; s. dazu auch TAYLOR 2010, 222223.

3 S. z.B. Assmann 2001, 528.

4 Vgl. Baines, Lacovara 2002; CoOney 2007, 275 und 282. Eine methodologische Betrachtung zu Gräberanalysen in der Ägyptologie liegt mittlerweile als Dissertation vor: ARP 2009.

5 S. beispielsweise GRAJETZKi 2003; SeIdLMAYER 2003.

6 Vgl. allgemein BernBEck 1997, 264. Der Eindruck, dass die Toten sich selbst begraben, wird allerdings in der Elitekult Ägyptens sehr bewusst durch die Bilder und Texte vermittelt; hier besteht ein großer Gegensatz zur Grundschichtkultur, s. SeIDlmayer 2003, 73 und CooNEY 2007, 276. 
heiten und Einzelfälle bei Bestattungsvorgängen in erster Linie weniger ein Zeichen geänderter Bestattungssitten, als vielmehr das Resultat einer möglichen Diskrepanz zwischen Theorie (Idealvorstellung) und Praxis, die durch subjektive Verhaltensweisen und Wahrnehmungen entstehen kann. ${ }^{7}$ Die Usurpation von Gräbern und Gegenständen, die Behandlung der Leichen oder singuläre Grabbeigaben könnten dafür Zeugnis ablegen. Denn stellt man das archäologische Fundmaterial, also die Objekte, in den Vordergrund, so wird selbst bei Relikten der Elitekultur ein „Vielerlei der gelebten Wirklichkeit"8 ${ }^{\text {hin- }}$ sichtlich der Bestattungen sichtbar. ${ }^{9}$

Grabbrauchtum und Bestattungsvorgänge sind somit auch innerhalb der Ägyptologie als sehr vielschichtig erkannt worden. Die Aufmerksamkeit wurde zunehmend auf die spezifischen Prozesse gelenkt, die zur Formation des spezifischen Befundes innerhalb der Gräber beitragen. In der Regel bietet ein altägyptisches Grab Funde aus unterschiedlichen Perioden und Relikte einer Vielzahl an Nutzungsphasen. Die Geschichte eines Grabes konstituiert sich folgerichtig aus der Summe verschiedener Fragmente unterschiedlicher Nutzungen durch Gruppen von Personen aus mehreren Phasen. ${ }^{10}$

In der ägyptischen, v.a. der thebanischen, Archäologie haben sich, aufbauend auf die theoretischen Überlegungen von Michael Schiffer zur Formation archäologischer Prozesse, ${ }^{11}$ in der Beschreibung der Nutzungsphasen von Gräbern die Begriffe use-life (deutsch "Gebrauchsleben“ und „Nachleben"12 oder auch „Lebensgeschich$\mathrm{te}^{\text {“13 }}$ ) sowie „systemische“ und „nicht-systemische“ Nutzungen eingebürgert. ${ }^{14}$ Der fallweise sehr gute Erhaltungszustand ägyptischer Grabbauten und die große Fülle an Artefakten und schrift- lichen Zeugnissen erlauben zuweilen eine minutiöse Rekonstruktion des use-life über lange Zeiträume - unterschiedliche Nutzungsarten und Nutzerkreise können dabei aufgezeigt werden. Die Palette der diesbezüglichen Möglichkeiten ist sehr groß und reicht von Wiederbestattungen und Kultbetrieb über Verfall, Beraubung und Restaurierung bis zur Zerstörung, profanen Zweckentfremdung oder Heiligenverehrung. Dabei stellt sich die Frage, inwieweit individuelle Einzellösungen oder aber regelhafte Verhaltensweisen, eventuell gebunden an das soziale Umfeld der Betroffenen, zum Tragen kamen.

In den meisten Fällen gilt das Hauptaugenmerk bei der Bearbeitung ägyptischer Grabbauten den Bestattungen aus der ersten Nutzungsphase, also der Zeit der Erbauung der Anlage. Spätere Ergänzungen und Wiederbelegungen werden zuweilen nur kursorisch erwähnt. So kommt es auch in Grabpublikationen zu einer gängigen, oft unbewussten und pauschalen Wertung von "primären" Bestattungen als hochwertiger und informativer gegenüber "sekundären“, und von intrusiven als weniger wichtigen und „ärmeren“ Grablegungen. ${ }^{15}$ Besonders in Veröffentlichungen $\mathrm{zu}$ thebanischen Gräbern des Neuen Reiches hat es sich aber durchgesetzt, dass neben den Funden aus der Erbauungsphase auch spätere Nachbestattungen vorgelegt werden. ${ }^{16}$ Sekundäre Ergänzungen, Umbauten und Wiederbestattungen werden berücksichtigt, wenn auch der Umfang, der dabei den „sekundären“ Funden eingeräumt wird, meist hinter denen der Primärbestattungen zurücksteht. ${ }^{17}$

Obwohl lange unterschiedliche Grabnutzungen durchgängig unter dem Begriff Usurpation subsumiert wurden, so gibt es seit den 1990er Jahren auch Untersuchungen zu häufigen Fällen
7 Vgl. BUDKa im Druck.

8 Seidlmayer 2003, 73.

9 Vgl. Baines, Lacovara 2002, 5-36.

10 Vgl. Guksch 1995.

11 SCHIFFER 1972; SCHIFFER 1987; vgl. jüngst auch eine gute Adaption seines Modells bei Hurcombe 2007, 39 43, Abb. 3.1.

12 Assmann 2005, 33.

13 Vgl. SeILER 2005, 21.

14 Nach Schiffer 1972; s. bes. POLz 1987; GUKSCH 1995; JÁNOSI 2005, 37, Anm. 29.

15 So hat z.B. WinLOCK 1932, zwar im Vorwort die Wichtigkeit der Präsentation sämtlicher Nutzungen erkannt, doch im Folgenden nimmt die Primärbestattung 45 Seiten des Textteils und 12 Seiten des Katalogs ein, während die spätere Nutzungsphase in 20 Textseiten und 2 Katalogseiten erwähnt wird. Dennoch ist diese Publikation hervorragend geeignet, um die Komplexität von Vorgängen rund um Beraubung, Restauration und Wiederbenutzung eines Grabes zu illustrieren.

16 S. z.B. Strudwick 1996; als ein Beispiel, dass in jüngster Zeit intrusiven Bestattungen auch mehr Raum bis hin zu unabhängigen Monographien gewidmet wird, s. SCHREIBER 2008.

17 S. z.B. SEYFrIEd 1990; SEYFrIEd 1995. 
geregelter, legaler Wiederbenutzung aus unterschiedlichen Beweggründen (v.a. Familien- oder Berufsbezogenheit sowie Namensgleichheit), ${ }^{18}$ gleichzeitiger Nutzung (Tomb sharing) ${ }^{19}$ aber auch zu tatsächlich unrechtmäßigen Aneignungen (Usurpationen), wobei der jeweilige Aufwand bei der Neugestaltung von Gräbern als stark variierend beobachtet wurde. ${ }^{20}$

Die meisten Studien zu Bestattungsabläufen und den Nutzungsarten von Gräbern basieren auf Befunden aus Gräbern des Neuen Reiches. Die Art der Grabnutzung ändert sich jedoch grundlegenden nach dieser Periode und unterscheidet sich v.a. in dem Anspruch, ein eigenes Grab zu errichten. ${ }^{21}$ Intrusive Schachtgräber in bestehenden Anlagen und Gruppenbegräbnisse sind die vorherrschenden Bestattungsformen der Dritten Zwischenzeit. ${ }^{22}$ Wurden in der 25. und 26. Dynastie wieder neue, innovative Anlagen geschaffen, ${ }^{23}$ so scheint es der Elite der altägyptischen Gesellschaft seit dem späten 6 . Jahrhundert v. Chr. neuerlich zu genügen, sich einen Platz in einem Grab zu sichern oder auch ein „fertiges" Grab zu kaufen. ${ }^{24}$ Dabei ist die „Usurpation“ eines Grabes nicht direkt proportional zum sozialen und wirtschaftlichen Hintergrund der Nutzer, sondern eher Ausdruck verschiedener Faktoren, die auch Veränderungen der funerärer Kultur umfassen. ${ }^{25}$

\section{Nachbestattungen in spätzeitliche Grabbauten in Theben}

Seit der ersten intensiven Beschäftigung mit den spätzeitlichen Tempelgräbern im Asasif in den 1960er Jahren ist bekannt, dass diese besonderen Bauten monumentalen Ausmaßes im großem Stil im 4., 3. und 2. Jahrhundert v. Chr. wiederverwendet wurden. ${ }^{26}$ Die archäologischen Befunde

\footnotetext{
18 Vgl. Polz 1990; Fitzenreiter 1994; KampP 1996, 123129.

19 Guksch 1995.

${ }^{20}$ SPENCER 1982; KAMPP 1996, 123-129.

${ }^{21} \mathrm{Zu}$ diesem Anspruch s. GUKSCH 1995.

${ }^{22}$ Vgl. Dodson, Ikram 2008, 270-273; Aston 2009, 398 und passim; TAYLOR 2010.

23 Zur Architektur s. Eigner 1984 (Theben), GESTERMANN 2006 (Memphis), Stammers 2009 (Memphis).

24 Aston 2003, 157.

25 BUDKA 2006, 794-796.

26 S. Assmann 1968, 22; de Meulenaere 1989, 55-73; Aston 2003, 162; Strudwick 2003, 172-174; BUdKA 2010a, 82-84.
}

werden dabei durch Textzeugnisse aus dem Umfeld der Archive der Choachyten ergänzt unter der Bezeichnung hw.t tauchen die Anlagen als über mehrere Generationen benutzte, kollektive Bestattungsplätze in diesbezüglichen Texten auf. ${ }^{27}$ Diese Blütephase späterer Nutzung ist nicht nur für die monumentale Elitegräber greifbar, sondern auch für benachbarte, kleine Anlagen desselben Zeithorizonts belegt. ${ }^{28}$

Im monumentalen Grab des Anch-Hor, TT 414, hat die späte Nutzung der Anlage zahlreiche Spuren hinterlassen und ist hinsichtlich Architektur, Dekoration und besonders durch Grabinventare gut greifbar. ${ }^{29}$ Darüber hinaus wurde in TT 414 das bislang einzige in situBegräbnis der 30. Dynastie in Theben sichergestellt - die Grabkammer des Wah-ib-Re mit ihren zahlreichen Funden. ${ }^{30}$

\subsection{Bestattungsabläufe im Spannungsfeld abstrakter Vorgaben und realer Umsetzung}

Ausgangspunkt der folgenden Überlegungen ist die These, dass insbesondere die Wiederverwendung von Gräbern den tatsächlichen, in erster Linie rationalen Umgang von Lebenden mit Toten illustriert, der sich als reale Praxis von idealisierten oder abstrakten Vorstellungen, wie sie sich etwa aus Textquellen und bildlichen Darstellungen erschließen, unterscheiden kann. ${ }^{31}$ Das Benehmen gegenüber und die Wertschätzung von Verstorbenen zeigt die alltäglich-praktische Seite des Totenkults von Seiten der Lebenden auf und ist in der Regel „ambivalent“. ${ }^{32}$ Tote wurden generell im alten Ägypten zum einen als hilfreiche Ahnen verehrt, aber auch zum anderen als bedrohliche Geister gefürchtet. ${ }^{33}$ So ist es wohl kein Zufall, dass sich der archäologische Befund

$\overline{27}$ Pestman 1993, 467. hw.t, $\square \square$, ist seit der 18. Dynastie als gelegentliche Bezeichnung für ein Grab belegt, s. Wb III, 2.10. Ob das ḥw.t der ptolemäischen Texte auch auf den Tempelcharakter der Monumentalgräber anspielt, ist deshalb nicht zu entscheiden.

28 S. BUDKA 2006, passim; BUDKA 2007; BUdKA 2010a.

29 Bietak, Reiser-Haslauer 1978, 78-85 und 141-151; BieTAK, Reiser-Haslauer 1982, 183-220.

30 BietaK, Reiser-Haslauer 1982, 183-220; Aston 2003, 162.

31 Vgl. Baines, Lacovara 2002.

32 Assmann 2005, 27; vgl. auch FITZENREITER 1994.

33 Vgl. TAYlor 2010, 221. Zu den unterschiedlichen Vorstellungen von Tod s. auch Assmann 2001, passim und bes. $89-115$. 
insbesondere rund um Beraubung, Zerstörung und Usurpation ebenfalls zwiespältig präsentiert. Abstrakte Ansichten des Totenkults und moralische Ideale der Elite wurden realpraktisch oft modifiziert gehandhabt oder gar missachtet, ${ }^{34}$ wofür sich v.a. im Nachleben der Grabanlagen Indizien finden lassen. Darüber hinaus sind spätestens ab der "Industrialisierung“ des Grabbrauchtums in der Spätzeit und der Ptolemäerzeit auch wirtschaftliche Aspekte zu berücksichtigen: Mumien waren essentielle Einkommensquellen für die Choachyten und wurden vererbt, verkauft und gehandelt. ${ }^{35}$

\section{Spätzeitliche Tempelgräber: Zeitgeist und Individualität}

Wesentliche kulturgeschichtliche Aspekte der Spätzeit, die sich auch auf die Konzeption der Grabanlagen ausgewirkt haben, sind zum einen Rückbezüge auf „archaische“ Architektur, Dekoration und Texte und zum anderen eine zunehmende Auffassung von Totenkult als Götterkult und eine Fokussierung auf Osiris und osirianische Aspekte. ${ }^{36}$ Der den Monumentalgräbern im Asasif innewohnende Tempelcharakter wird wohl dafür verantwortlich sein, dass man Teile des Grabes der Öffentlichkeit zugänglich machte und durch eine gewisse Unfertigkeit der Anlagen nachfolgende Ergänzungen als erwünscht deklarierte. ${ }^{37}$ So fordert beispielsweise der Grabherr Ibi im 6. Jahrhundert v. Chr. zukünftige Besucher seines Grabes (TT 36) auf: „Wo immer ihr wollt, da schreibt auf die freigelassenen Stellen! ${ }^{* 38}$ Hierbei scheint es sich eher um eine Erscheinung des spezifischen Zeitgeists zu handeln, als um eine individuelle Note des Grabherrn. ${ }^{39}$

34 Vgl. Baines, LaCOVARA 2002, 23; COONEy 2007, 273-275; FITZENREITER 2008.

35 Pestman 1993, 444. EbD., 101-102 gibt es Hinweise für das Verständnis von Mumien als Einnahmequellen, und zwar in einer Beschwerde über eine Grabplünderung (no. 3, Louvre 2330, 127/26 v. Chr.). Dem Text zufolge wurde ein "waiting-tomb" eines Choachyten geplündert, also eine temporäre Lagerstelle von Mumien und Geräten, wobei sowohl Leichen als auch die wertvolle Ausstattung des Totenpriesters den Dieben zum Opfer viel. Die Beschwerde betont die finanzielle Seite des Verlusts, wobei die Geräte an erster Stelle stehen.

36 Vgl. BUdKA 2010a, 488 und passim.

37 Bereits im Neuen Reich waren thebanische Felsgräber teilweise zugänglich und besonders in der Ramessi-
Da Bestattungsabläufe zwangsläufig in Beziehung zur Architektur und Dekoration von Gräbern stehen, können auch diese in einem ersten Schritt auf ihre Regelhaftigkeit geprüft werden. Bei den thebanischen Tempelgräbern der Spätzeit ist es deshalb auffallend, dass sich die Anlagen aufgrund einer großen Variantenbreite an Bauformen nicht in einem Idealtyp definieren lassen. Es gibt zwar generelle Richtlinien und wichtige Bauelemente, aber wie diese kombiniert und im Detail ausgeformt wurden, ist von Grab zu Grab unterschiedlich. ${ }^{40}$ Offenbar konnten also die höchsten Beamten, insbesondere die Obersthofmeister der Gottesgemahlinnen, ihre Gräber nach individuellen Vorlieben gestalten - dies gilt sowohl für die Architektur, als auch die Dekoration. ${ }^{41}$ Darüber hinaus müssen die jeweiligen topographischen Gegebenheiten und Platzverhältnisse beim Bau der Anlage berücksichtigt werden. Auch die Nähe zu älteren Anlagen aus dem Neuen Reich hat sich teilweise stark ausgewirkt, wurden doch Szenen aus Vorgängerbauten kopiert. ${ }^{42}$

Das Maß an Individualität, das die höchsten Beamten der Spätzeit bei der Konzeption ihrer Grabpaläste im Asasif einbringen konnten, scheint aber weniger eine rein zeitgemäße Erscheinung und Ausdruck der innenpolitischen Machtverhältnisse der damaligen Zeit zu sein, als vielmehr ein Privileg einflussreicher Personen, wie es sich ähnlich schon früher, besonders in der Ramessidenzeit, nachweisen lässt. ${ }^{43}$

Lohnenswert präsentiert sich nun die Fragestellung, ob sich ähnlich wie bei Architektur und Dekoration auch in den Grabbeigaben, in der Grablegung selbst, individuelle Züge finden lassen. Dem steht zwar vorderhand der fragmentari-

denzeit ist ein tempelähnlicher Charakter für die Vorhöfe festzustellen, s. u.a. KAMPP 1996, 118-119. Ähnliches gilt auch für die Elitegräber im memphitischen Raum, die ebenfalls immer mehr Züge eines Tempels annehmen, vgl. KITCHEN 1979.

38 KuHLMANn 1973, 210.

${ }^{39} \mathrm{Zu}$ unterschiedlichen Theorien möglicher Wechselbeziehungen zwischen „Archaismus“ und Individualität s. NEUREITER 1995.

40 Eigner 1984, 59-61.

${ }^{41}$ S. z.B. die Besonderheiten in TT 27, Roccati 1995.

42 Vgl. Bietak, in: BietaK, Reiser-Haslauer 1982, 232-240.

43 Vgl. KAMPP-SEYFried 2003, 126 (singuläre, individuelle Bildschöpfungen); GNIRS 2003, 179-187 (singuläre Textkreationen). 
sche Erhaltungszustand der Grabinventare aus den Monumentalgräbern entgegen, doch gestattet, wie im Folgenden gezeigt werden soll, die umfassende Analyse der Bestattungsabläufe einige Beobachtungen. Wesentliches Potential liegt insbesondere im Studium der Nachnutzung der Tempelgräber und der diachronen Entwicklung ihrer Belegung. Ob variierende Bestattungsabläufe auf individuelle Vorlieben und Entscheidungen zurückgehen, oder die praktische Umsetzung abstrakter Regelsysteme mit einem gewissen Handlungsspielraum sind, gilt es zu untersuchen.

\section{Fallbeispiel TT 414:}

\section{Ausgewählte Bestattungen der Hauptnut- ZUNGSPHASEN}

Das aktuelle Anch-Hor-Projekt hat sich zum Ziel gesetzt, die Bestattungsabläufe und Grabinventare in TT 414 in ihrer Gesamtheit zu erfassen. ${ }^{44}$ Die Grabstätte von Anch-Hor blieb beim Tod seines Erbauers (585 v. Chr.) noch unfertig, wurde in weiterer Folge mehrfach verändert, erweitert, zerstört, restauriert und in hohem Maße geplün- dert. Die allgemeine Nutzung dauerte bis in spätrömische Zeit und hat vielfältige archäologische Spuren hinterlassen. Im Folgenden wird lediglich eine Auswahl von Funden der 26. Dynastie bis in die ptolemäische Epoche vorgestellt. Zur Rekonstruktion der vollständigen Belegungsphase sind noch dringend weitere Detailstudien an den Objekten notwendig, die in der nahen Zukunft erfolgen sollen.

\subsection{Bestattungsabläufe der 26. Dynastie: Die Kernfamilie des Anch-Hor}

TT 414 war, wie alle derartige Monumentalgräber im Asasif, als Familiengrab konzipiert und etliche Verwandte wurden bei Anch-Hor mitbestattet (z.B. eine Tochter, mehrere Brüder, eine Schwester).$^{45}$ In der ersten Bauphase umfasst die unterirdische Kultanlage drei Nebenräume (Raum 7, 8, 9) im hinteren Bereich des Grabes, von denen jeweils Schachtanlagen zu den Bestattungsanlagen führten (Fig. 1). In den Grabkammern am Boden dieser Schächte waren Mitglieder der Familie des Anch-Hor bestattet. Enge Verwandte,

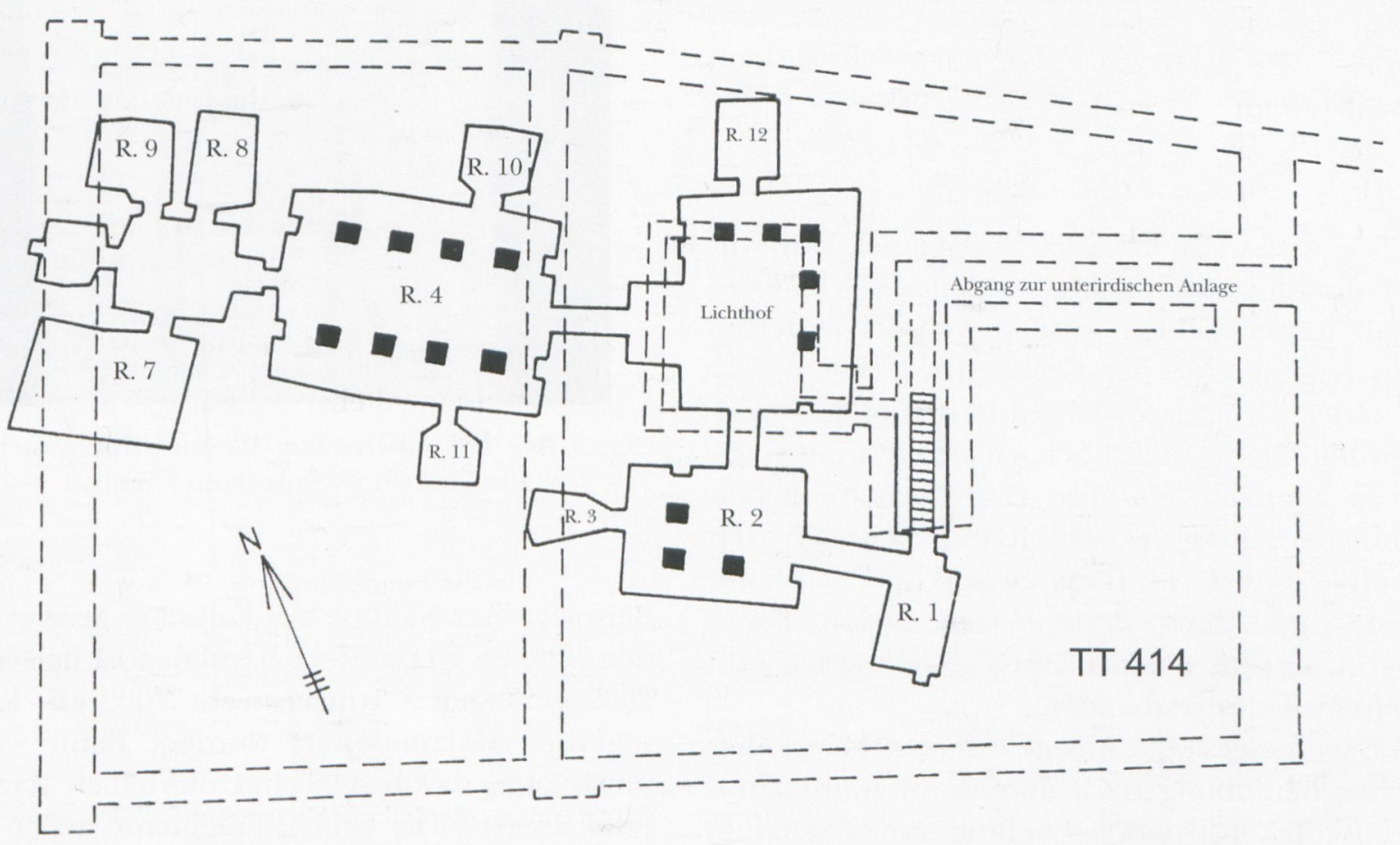

Fig. 1 TT 414, Plan der unterirdischen Kultanlage (nach: M. BietaK, E. Reiser-HasLauer 1979, Plan 10) ${ }^{44}$ Vgl. BUDKA 2008a; BUDKA 2008b; BUDKA 2009a; BUDKA
2009b; BUDKA 2010b. 45 Bietak, Reiser-Haslauer 1982, 176-178, 250-251;
Budka 2008a, 66-68, Fig. 4 . 


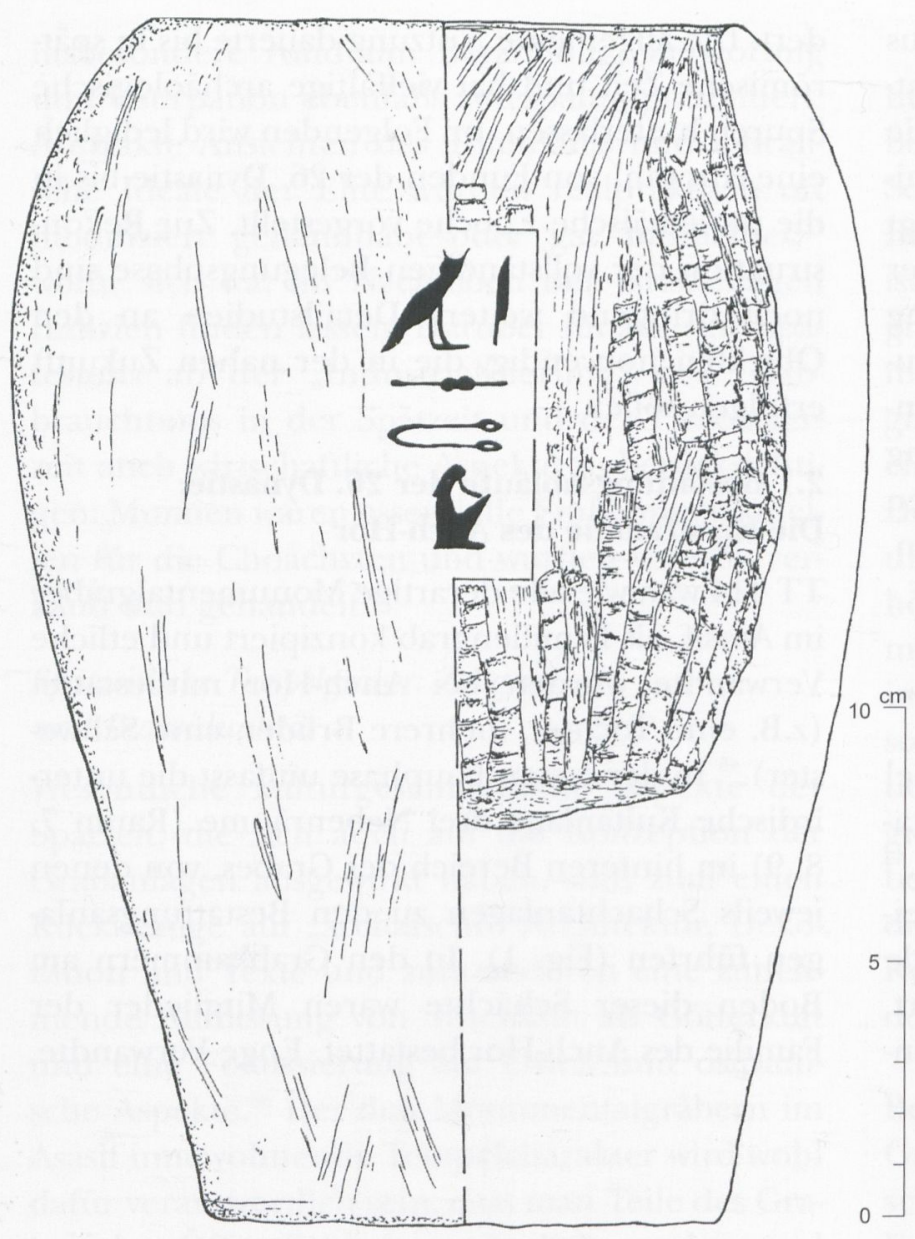

Fig. 2 Reg. 08/02, Kanope aus Kalkstein

(Originalzeichnung N. Richter, Umsetzung J. Budka)

wie beispielsweise die Ehefrau des Anch-Hor, von der allerdings noch immer eindeutige Relikte des Grabinventars fehlen, wurden eventuell in derselben Kammer wie der Grabbesitzer (Raum 7.1) bestattet, für andere wurden in den nahe liegenden Räumen 8 und 9 Schachtanlagen mit Kammern ausgemeißelt. Ihre Grabinventare haben ähnliche Schicksale erlitten und befinden sich heute sämtlich in fragmentarischem Zustand. Reste von Särgen, Perlennetzen, Stelen, Kanopen, Uschebtis, Ptah-Sokar-Osiris-Statuetten und Schreinen sind vorhanden.

Dass die gesamte Anlage offenbar bei wiederholter Beraubung stark durchwühlt wurde und bei der Rekonstruktion von Bestattungsorten aufgrund der Fundverteilung des Grabinventars entsprechende Vorsicht gelten muss, ${ }^{46}$ mag mit dem

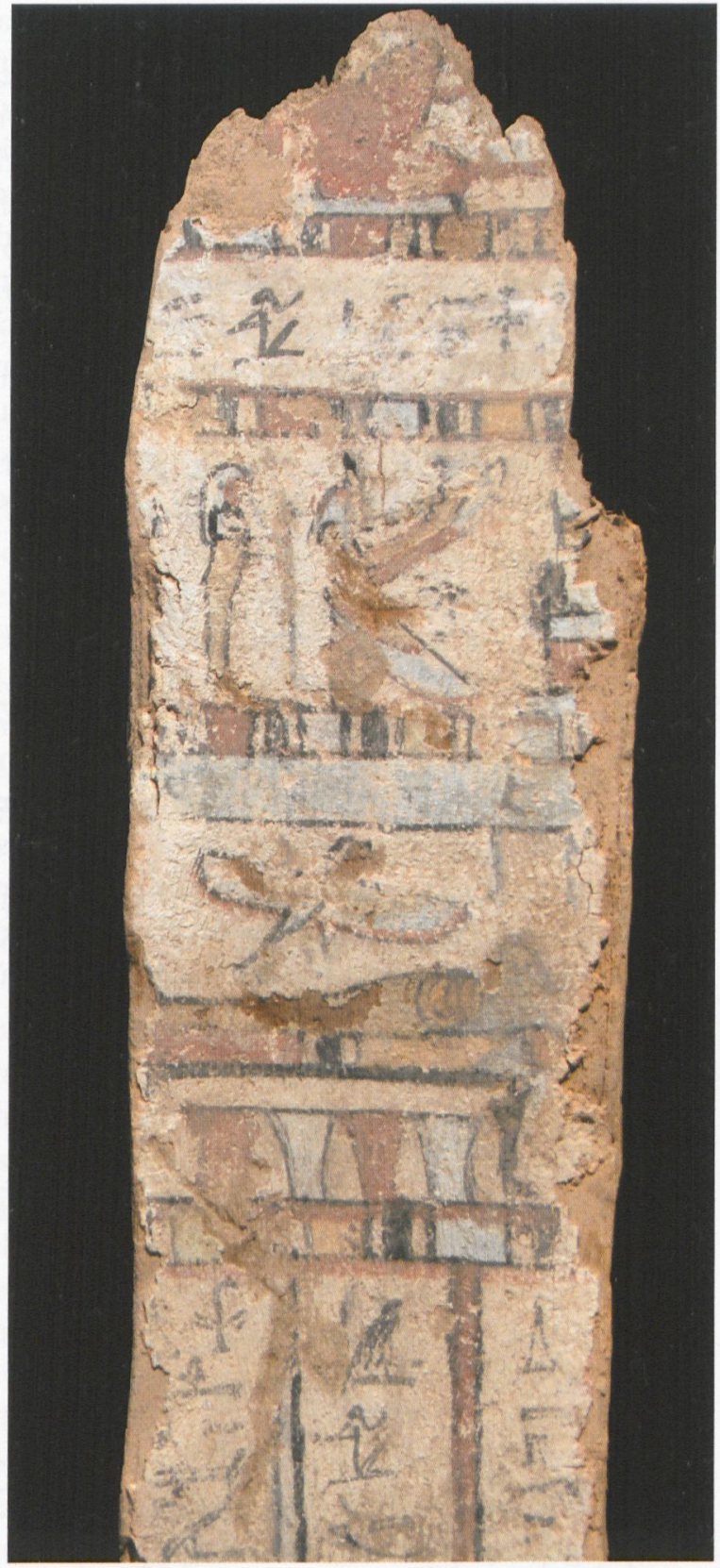

Fig. 3 Reg. 537, innerer Sarg des Anch-Hor: Ausschnitt des Deckelfragments (Foto J. Budka)

Beispiel einer Kanope aus Kalkstein gezeigt werden (Fig. 2). Deren Rekonstruktion ist im Herbst 2009 gelungen - zunächst war 2007 ein Randscherben dokumentiert worden, dann wurde unabhängig davon 2008 ein Unterteil im Magazin relokalisiert. ${ }^{47}$ Die beiden Fragmente ließen sich nun neu aneinander passen und so liegt das vollständige Profil einer wohl saitischen Kanope vor.

\footnotetext{
${ }^{47}$ Dieser Unterteil wurde bereits von BIETAK, REISER-HASLAUER 1982, 179, erwähnt.
} 


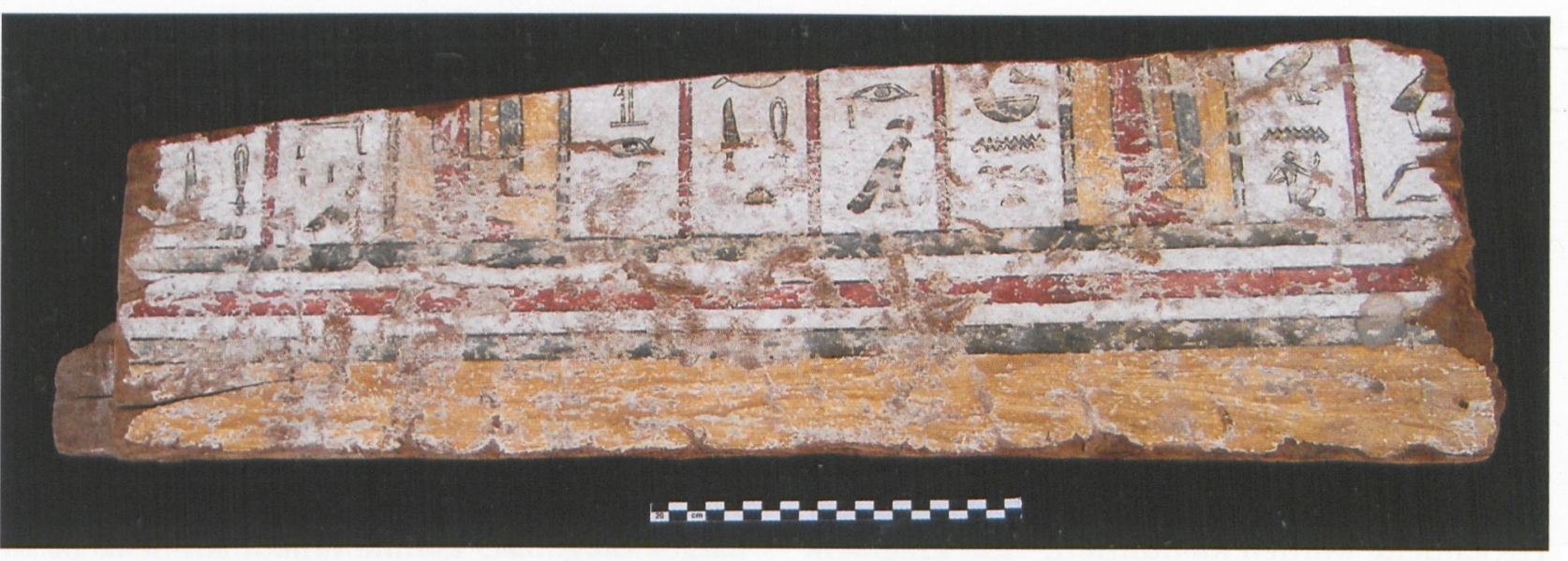

Fig. 4 Reg. 519, Haussarg der Her-aset: Fragment der Längsseite (Foto J. Budka)

Die Tintenaufschrift auf der Schulter gibt den Namen des Horussohnes Amset wieder. Wie es insbesondere für Scheinkanopen der Dritten
Zwischenzeit üblich ist, aber wie es sich auch in der Spätzeit für reale Kanopen nachweisen lässt, ${ }^{48}$ wird ein Besitzernamen nicht genannt.

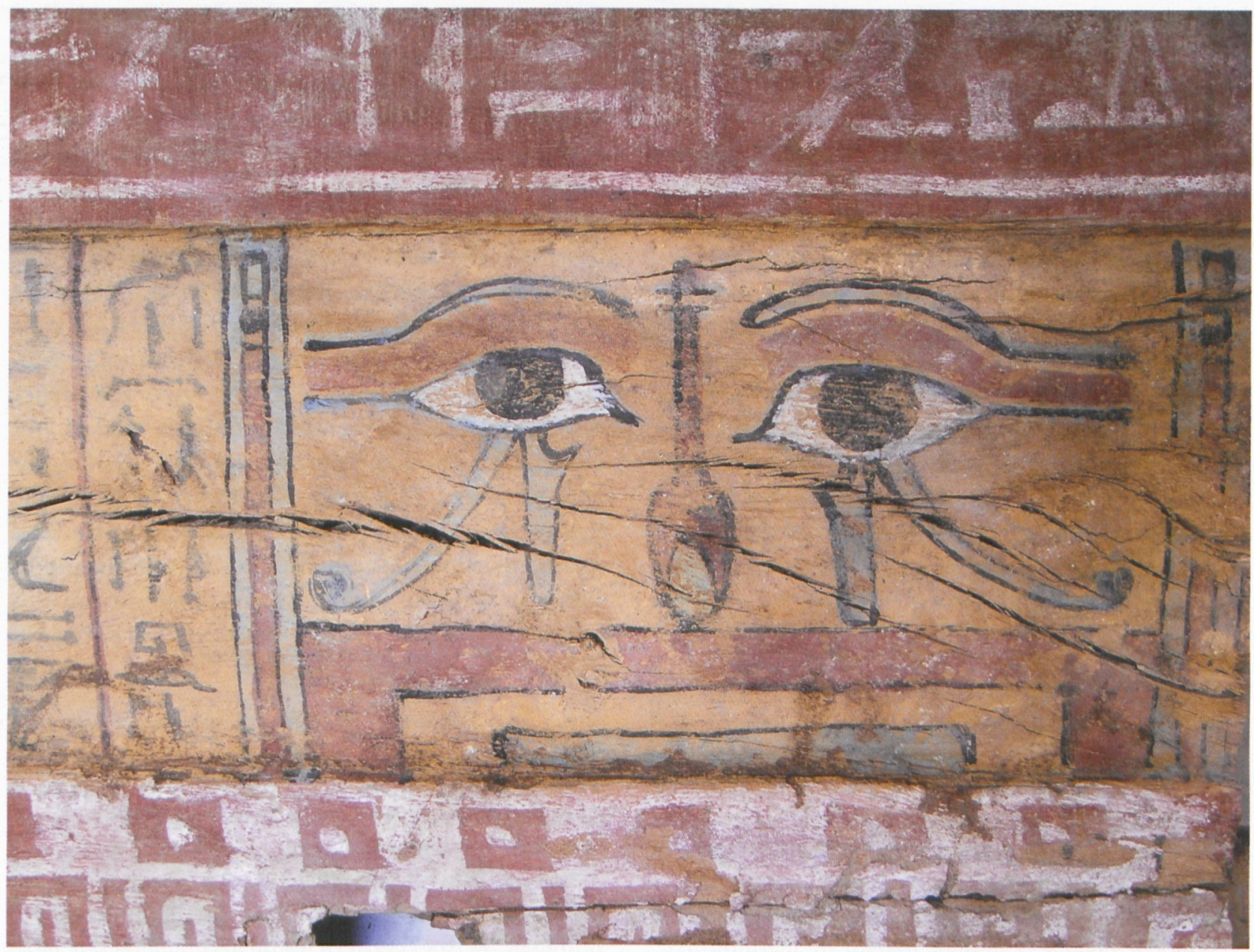

Fig. 5 Reg. 595, Pfostensarg des Psammetich-men-em-Waset II: Detail der Längsseite (Foto J. Budka)

48 Vgl. beispielsweise Brovarksi 1978, 13-16 (Boston MFA 72.586-589), 17-20 (Boston MFA 72.590-593) (Dritte Zwischenzeit) und 37-38 (Boston MFA 72.613, Spätzeit). 
Die Fundposition der Bruchstücke ist nun beispielhaft für die generell weite Streuung der Objekte in TT 414: Das Unterteil stammt aus dem hinteren Bereich, dem Schacht von Raum 8; das Randstück hingegen aus dem Eingangsbereich zur unterirdischen Anlage (vgl. Fig. 1). Einige registrierte Stücke aus dem entsprechenden Schacht 8 können übrigens ebenfalls in die 26. Dynastie datiert werden - so die Reste des Grabinventars der Her-aset, möglicherweise einer Schwägerin des Anch-Hor: ein anthropoider Sarg, eine Holzstele und eine Ptah-Sokar-Osiris-Statuette wurden identifiziert. ${ }^{49}$ Ein Fragment des zugehörigen Haussarges (Reg. 519, Fig. 4) kam in Raum 4 zutage und illustriert die qualitativ hochwertige Ausführung der Grabbeigaben. $\mathrm{Ob}$ nun auch die neu rekonstruierte Kanope einst Bestandteil dieses Ensembles war, muss offen bleiben.

Fragmentarisch auf uns gekommen sind die beiden anthropoiden Särge des Anch-Hor. Besonders der innere Holzsarg (Reg. 537, Fig. 3) ${ }^{50}$ fällt durch eine sehr flüchtig ausgeführte Bemalung geringer Qualität auf - ist dies allein auf den verfrühten Todes des Grabherrn zurückzuführen, wie auch der unfertige Zustand der unterirdischen Kultanlage? Zeitmangel, Kostenersparnis oder einfach mangelhafte Ausführung sowie geringe Wertigkeit - war der innere Sarg doch kein „sichtbares“ Prestigeobjekt - sind die wohl wahrscheinlichsten Rahmenbedingungen rund um Reg. 537, doch der reale Ablauf seiner Produktion lässt sich nicht mit Sicherheit rekonstruieren. ${ }^{51}$ Festzuhalten bleibt, dass dieser Holzsarg im starken Gegensatz zur Architektur, Größe und Dekoration der Anlage steht und sich v.a. auch von den nahezu gleichzeitigen Särgen der Verwandten des Anch-Hor unterscheidet (s. Fig. 4 und 5).

49 Vgl. Bietak, Reiser-Haslauer 1982, 178 und 279, G 111; ein großer Teil der Holzstele befindet sich heute im British Museum London, s. EBD., Taf. 155 (BIERBRIER 1987, 23, Taf. 38-39).

50 BietaK, Reiser-Haslauer 1982, 167-170, Abb. 69, 70, 73 und Taf. 93.

51 Für gut belegte Vorgänge, Preis- und Wertvorstellungen rund um ramessidische Särge s. COONEY 2007, passim.

52 Bietak, Reiser-Haslauer 1982, 182, 250-251 und 277, G 86 .

53 BietaK, Reiser-Haslauer 1978, 141; vgl. BudKa 2009a, 83.
Vergleichsweise gut erhalten und von hoher Qualität sind beispielsweise der Pfostensarg und der anthropoide innere Sarg eines Verwandten des Anch-Hor namens Psammetich-men-em-Waset II. (möglicherweise sein Neffe oder Enkel)..$^{52}$ Die Bretter des Haussarges (Reg. 595, Fig. 5) wurden an unterschiedlichen Stellen des Grabes entdekkt: die größten Fragmente stammen aus dem stark durchwühlten Schacht von Raum 10, doch ein weiteres Brett wurde als Architrav verbaut in einer Ziegelabmauerung des „Lichthofes“ aufgespürt. ${ }^{53}$ Die Wanne des dazugehörigen inneren Sarges (Reg. 591) konnte erneut aus Schacht 10 geborgen werden. Die ehemals aufwendige Bemalung auf Leinwand, für die sich zahlreiche Parallelen finden lassen, hat aufgrund der Beraubung und Umlagerung stark gelitten. ${ }^{54}$ Der schlechte Erhaltungszustand des gesamten Sargensembles ist also sowohl auf eine wiederholte Beraubung als auch die Wiederverwendung und damit zusammenhängende Baumassnahmen zurückzuführen.

Einige bemalte Holzstelen der 26. Dynastie kamen in TT 414 zutage und wurden bereits an anderer Stelle besprochen. ${ }^{55}$ Die vollständige Stele Reg. 506, KhM A2096 $6^{56}$ sei hier jedoch erwähnt. Das Stück kam im Schacht von Raum 10 in $5 \mathrm{~m}$ Tiefe der Verfüllung zum Vorschein und könnte eine saitische Bestattung in Grabkammer 10.1 belegen. Der Name des Besitzers ist zwar nicht mehr lesbar, aber aufgrund des Titels „Kammerherr der Gottesverehrerin" (jmj-hnt $n$ dw3.t-ntr) wird es sich um ein Familienmitglied des AnchHor gehandelt haben. ${ }^{57}$ Möglicherweise war der Stelenbesitzer ein enger Verwandter (Bruder/ Vater?) von Psammetich-men-em-Waset II., dessen Sargfragmente zum Teil auch aus Schacht 10 stammen. ${ }^{58}$ Beim jetzigen Kenntnisstand wäre es möglich, dass die Schachtoberräume im vorderen

${ }^{54}$ Für Vergleichsbeispiele derartiger Särge s. TAYLOR 1989, 59, Abb. 48; TAYLOR 2003, 115, Taf. 67.

55 BUDKA 2008a, 68-69.

56 SAtZinger 1979, 106-107, M 4 (Abb. 95); BUdKA 2006, Bd. IV, 191-192, Kat. 497.

57 Bietak, Reiser-Haslauer 1982, 183. Für diesen möglichen Sohn (oder Schwiegersohn) des Anch-Hor s. auch BIETAK, REISER-HASLAUER 1982, 251.

58 Hinzuweisen ist jedoch an dieser Stelle, dass auch Sargfragmente von Anch-Hor, Reg. 537, aus eben diesem Schacht stammen, der Befund also stark gestört ist (s. BietaK, Reiser-HASLAuer 1982, 182). 
Bereich der Kulträume (Räume 10 und 11) von einem Nebenzweig der Familie des Anch-Hor nach den Schächten im rückwärtigen Trakt von TT 414 angelegt wurden. ${ }^{59}$ Die zeitliche Differenz mag dabei etwa eine Generation betragen haben.

\subsection{Ausgewählte Bestattungsabläufe der 30. Dynastie}

\section{Allgemeines}

Schon mehrfach wurde in der Forschung thematisiert, dass die monumentalen Spätzeitgräber im Asasif besonders während der 30. Dynastie und der Ptolemäerzeit (4. und 3.-2. Jahrhundert v. Chr.) erneut belegt wurden und insbesondere Familien, die Funktionen im Tempeldienst (Priester- oder Handwerkertätigkeiten) ausübten, als Gruppenbegräbnisstätten dienten.$^{60}$ Die Belegung der einzelnen Kammern scheint in ihrer Abfolge der horizontalen Sozialhierarchie innerhalb der Familien zu folgen. Dafür sprechen sowohl Textzeugnissen ${ }^{61}$ als auch archäologische Hinweise, wie die Bestattungsabläufe der Familie des Wah-ib-Re I. im Grab des Anch-Hor. ${ }^{62}$ Da die meisten der bestatteten Personen beruflich mit dem Tempel des Amun von Karnak verknüpft waren, dürfte auch die zweite Blütezeit des Asasifs im 4. Jahrhundert v. Chr. mit kulttopographischen Bezügen, der Tradition des Talfestes und der Nähe des Areals zu Deir el-Bahari im Zusammenhang stehen. ${ }^{63}$

Bereits vor der Ausgrabung durch Manfred Bietak in den 1970er Jahren, waren im Grab des Anch-Hor, TT 414, zahlreiche ptolemäische Särge, Stelen und Papyri gesichtet worden. ${ }^{64}$ Die beiden letztgenannten haben bereits im 19. Jahrhundert als leicht transportable Fundkategorien ihren Weg nach Europa gefunden. Eine Gruppe dieser Artefakte befindet sich heute im British Museum in London. ${ }^{65}$

Als Ort der sekundären Bestattungen kamen in erster Linie die originalen Schachtanlagen und Kammern zum Einsatz - doch auch neue, unabhängige Schachtsysteme, Erweiterungen und Kammern wurden aus dem Fels gemeisselt. Ähnliches scheint für die Begräbnisausstattung zu gelten: Neben neuen, individuellen Anfertigungen erfuhren auch Teile der ursprünglichen Grabinventare eine Wiederverwendung (siehe unten).

\section{Bestattungen der Kernfamilie des Padi-imen-neb- nesuttaui}

Die Bestattungsanlage von Anch-Hor selbst, Raum 7.1, wurde von einem Amunpriester namens Padi-imen-neb-nesuttaui I. (P3-dj-jmn-nb$n s w t-t 3 . w j)$ und seiner Familie wiederbenutzt. ${ }^{66}$ Davon zeugen polierte Naturholzsärge mit geschnitzter Dekoration sowie eine Gruppe bunt bemalter anthropoider Särge, die in der 30. Dynastie und der frühptolemäischen Zeit angefertigt wurden und dort eingebracht wurden. ${ }^{67}$

Für Wah-ib-Re I., einen Amunpriester und Sohn des Padi-imen-neb-nesuttaui I., ${ }^{68}$ wurde der bereits bestehende Schacht 10 erweitert und eine neue Kammer an dessen Sohle ausgehauen (Raum 10.2). Sein Grabinventar wurde als einziges intakt, wenn auch stark von Grundwasser in Mitleidenschaft gezogen, vorgefunden. Obwohl wir generell wenig über Grabbeigaben der 30 . Dynastie wissen, ${ }^{69}$ scheinen sich doch einige besondere, möglicherweise individuelle Details bei Wah-ib-Re I. feststellen zu lassen: Dies wäre zum einen die singuläre Mitgabe eines kompletten Sets an Balsamierungsbesteck, ${ }^{70}$ und zum
59 BietaK, Reiser-Haslauer 1978, 78 und BietaK, Reiser-HasLAUER 1982, 180 hielten die Räume 10 und 11 eher für sekundär. Mit ihrer geringen Tiefe und den einfachen, oberen Kammern erinnern beide aber an die der ersten Nutzungsphase zugeschriebenen Räume 8 und 9, weshalb hier eine frühere Datierung nicht ausgeschlossen wird.

60 Burkard 1986, 16; De Meulenaere 1989; Pestman 1993, 449. Zusammenfassend auch Aston 2003, 162-163 und STRUdwick 2003, 172-174 mit Abb. 3.

${ }^{61}$ Vleeming 1995, 251.

62 Bietak, Reiser-Haslauer 1982, 252-257.

63 Zur Bedeutung des Talfestes für die Entwicklungsgeschichte des Asasif s. BietaK, in: BIETAK, ReIser-HaslauER 1978, 19-29; vgl. auch BUDKA 2010a, 477-486.
64 Budka 2008a, 64-65 für frühe Beobachtungen zu TT 414, besonders durch LePsius.

65 S. Bierbrier 1987, 23, 30, 36-39; QUIRKE 1993, 21; zusammenfassend auch BUDKA 2008a, 69 und 75-77.

66 S. die rekonstruierten Genealogien bei MunRo 1973, 57-58; BietaK, REISER-HASLAuer 1982, 252-256.

67 Bietak, Reiser-Haslauer 1982, 166.

68 Ausführlich zu diesem Befund s. BIETAK, ReISER-HaSLAUER 1982, 199-220 sowie zusammenfassend GRAJETZKI 2003, 119-121.

69 Vgl. die idealisierte Rekonstruktion aufgrund des Befundes von Wah-ib-Re I. bei AsTon 2003, 162-163 und Abb. 18.

${ }^{70}$ BietaK, Reiser-Haslauer 1982, 186-192, Abb. 84. 


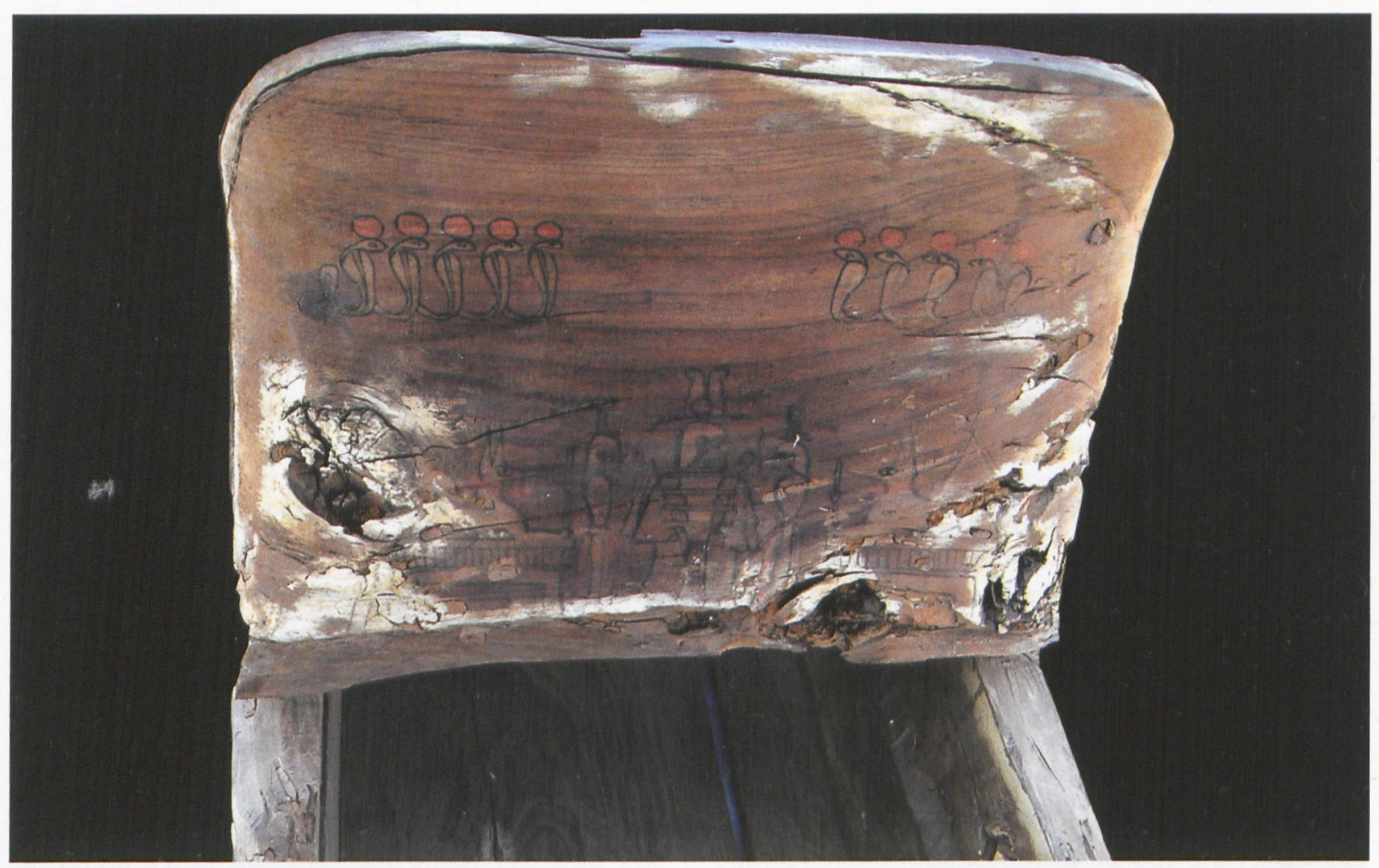

Fig. 6 Reg. 865, innerer Sarg des Wah-ib-Re I, Fußteil (Foto J. Budka)

anderen die ungewöhnliche Bemalung des Fußteils seines inneren Sarges (Fig. 6). ${ }^{71}$

Stärker als beim Grabinventar des Wah-ib-Re I., lassen sich bei Objekten aus der Grabkammer 7.1 Bezüge zur 26. Dynastie feststellen. Bemalte Särge wie derjenige eines Verwandten des Padiimen-neb-nesuttaui I. namens $P 3-d j-c_{-}$(Reg. 655) greifen beispielsweise viele Motive und Bildinhalte aus der Spätzeit auf und illustrieren so anschaulich einen thebanischen "Archaismus" hinsichtlich der Sargdekoration. ${ }^{72}$ Obwohl in Zusammenhang mit den vielfältigen Bezugnahmen auf ältere Stücke und Bildmotive noch reichlich Fragen offen sind, so hat es doch bei den Fallbeispielen aus TT 414 den Anschein, als hätte das Material der ersten Nutzungsphase als direkte Inspirationsquelle gedient. Man wollte sich offenbar nicht nur in einem saitischen Grab bestatten lassen, sondern vorzugsweise auch in der ursprünglichen Grabkammer des Besitzers zur letzten Ruhe kommen, und zwar ausgestattet mit „traditionellen“ Gegenständen. Wah-ib-Re I. ist hingegen eigene Wege mit persönlichen Besonderheiten gegangen.

Der Befund gewinnt dadurch an Komplexität, dass offenbar zeitgleich wie diese ,archaisierenden" Särge eingebracht wurden, auch originale Särge der 26. Dynastie demoliert und als Baumaterial im Lichthof oder in Torvermauerungen verbaut wurden. ${ }^{73}$ Ist dieses ambivalente Bild durch individuelle Entscheidungen bestimmter Personen oder unterschiedliche Personenkreise (Aufsicht bei der Instandsetzung des Lichthofs vs. Durchführung von Bestattungen) zu erklären? Oder waren die Särge bereits zerstört vorgefunden worden und wurden so nur noch als praktisches Baumaterial wahrgenommen?

\subsection{Bestattungsabläufe der Ptolemäerzeit}

Grablegungen der Familienmitglieder des $P 3-d j-$ jmn-nb-nswt-t3.wj I. fanden mindestens bis in die frühptolemäische Zeit hinein statt. Während des 2. Jahrhunderts v. Chr. und auch in spätptolemäischer Zeit kam es in TT 414 außerdem weiterhin

${ }^{71}$ Für die komplette Ansicht des Fußteils s. BIETAK, REISER-HASLAuer 1982, Taf. 136.

\footnotetext{
72 BUdKA 2008a, 71-72, Fig. 6.

73 S. BietaK, Reiser-Haslauer 1982, 180-182, Abb. 77.
} 


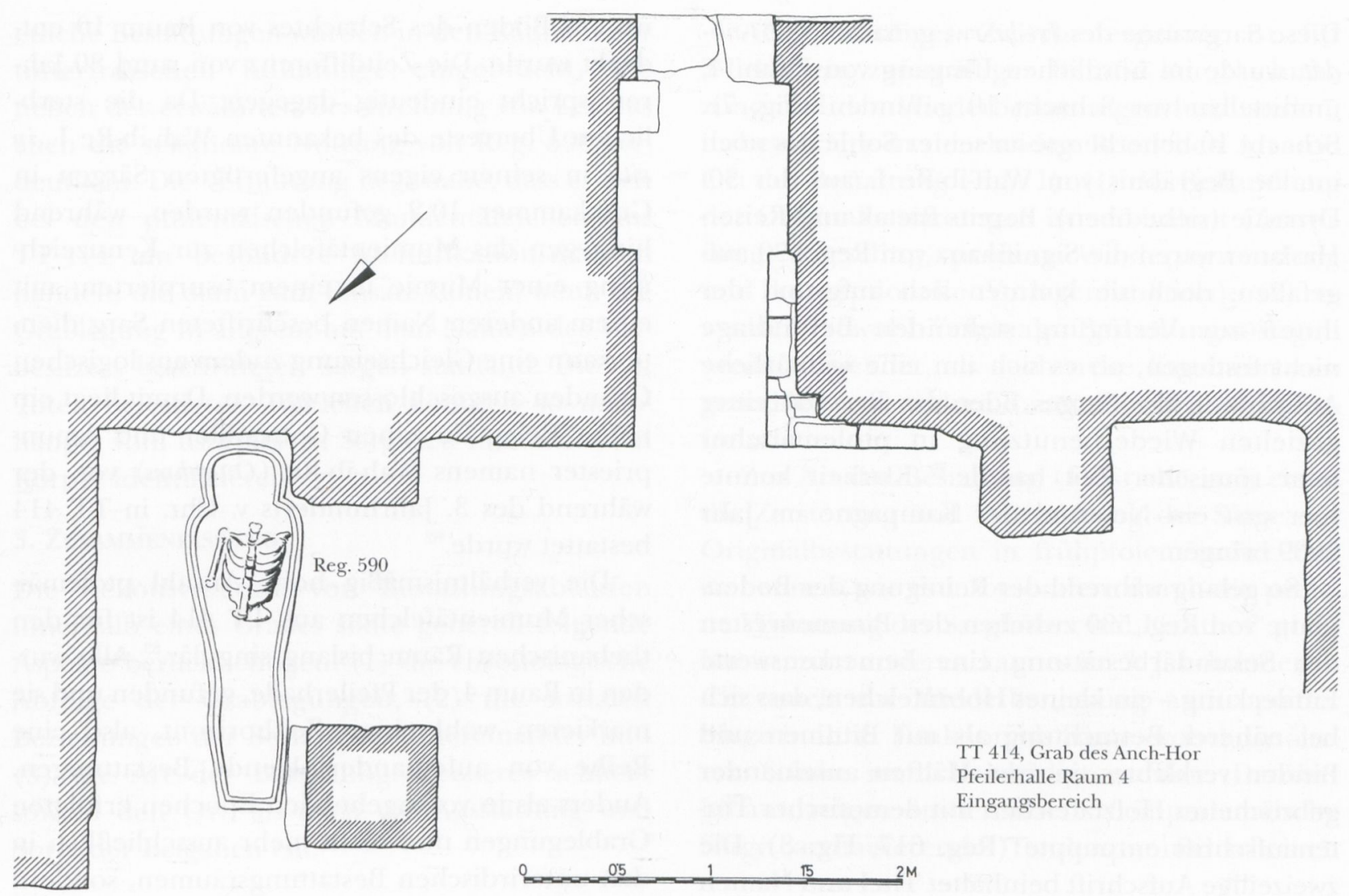

Fig. 7 In situ-Position von Sarg Reg. 590 in Pfeilerraum 4

(aus: M. BietaK, E. Reiser-Haslauer 1982, Abb. 64)

zu Bestattungen - Särge, Schreine, Statuetten und andere Grabbeigaben zeugen von diesen Begräbnissen. ${ }^{74}$

Spätestens jetzt wurden Bestattungen offenbar auch in der unterirdischen Kultanlage selbst eingebracht, also nicht mehr ausschließlich in den unterirdischen Bestattungsräumen. Dies wird besonders in der Beschreibung der Anlage durch Richard Lepsius deutlich (LD, Text III, 284): „,.. ein großes Psammetichgrab, das an 30 hölzerne Sarkophage, z.T. sehr schön vergoldete, einen neben den anderen aufgestellt enthielt ..." ${ }^{\text {"75 }}$ Als Aufstellungsplatz dieser Särge nennt Lepsius in erster Linie die große Pfeilerhalle (Raum 4), aber auch diverse Nebenräume (wohl die Räume 10, 11 und vielleicht 5 und 6 oder 7, vgl. Fig. 1). Aus der unterirdischen Kultanlage, besonders aber dem Lichthof, scheinen darüber hinaus einige der Holzste- len zu stammen, die sich heute u.a. in British Museum befinden. ${ }^{76}$

Recycling von Särgen

Wie oben erwähnt wurde, wurden va. Haussärge der 26. Dynastie als Baumaterial bei Instandsetzungen im Lichthof recycelt. ${ }^{77}$ Doch auch anthropoide Särge wurden in späteren Nutzungsphasen wiederverwendet. Anscheinend fand diese Form des Recycling dabei in ihrer Funktion als Särge statt - Indizien dafür sind intakte saitische Sargunterteile, die liegend in Raum 4, der großen Pfeilerhalle, gefunden wurden und ptolemäische Mumientäfelchen (3. Jahrhundert v. Chr.) aus demselben Raum. ${ }^{78}$

Ein konkretes Beispiel für eine ptolemäische Wiederverwendung stellt die Sargwanne eines inneren anthropoiden Sarges, Reg. 590, dar.

\footnotetext{
74 Vgl. BuDKa 2008a, 78.

75 S. Eigner 1984, 54; BUDKA 2008a, 64-65.

76 BUDKA 2008a, 75-78.
} 
Diese Sargwanne des Jrt-hr-rw, genannt Ns-b3-nb$\underline{d} d t$, wurde im nördlichen Umgang von Raum 4, unmittelbar vor Schacht 10 gefunden (Fig. 7). Schacht 10 beherbergte an seiner Sohle das noch intakte Begräbnis von Wah-ib-Re I. aus der 30. Dynastie (siehe oben). Bereits Bietak und ReiserHaslauer waren die Signifikanz von Reg. 590 aufgefallen, doch sie konnten sich aufgrund der ihnen zur Verfügung stehenden Befundlage nicht festlegen, ob es sich um eine willkürliche Anordnung des Sarges oder das Resultat einer gezielten Wiederbenutzung in ptolemäischer oder römischer Zeit handle. ${ }^{79}$ Klarheit konnte hier erst ein Neufund der Kampagne im Jahr 2009 bringen.

So gelang während der Reinigung der Bodenplatte von Reg. 590 zwischen den Bitumenresten der Sekundärbestattung eine bemerkenswerte Entdeckung - ein kleines Holztäfelchen, dass sich bei näherer Betrachtung als mit Bitumen und Binden verklebtes, in zwei Hälften auseinander gebrochenes Holztäfelchen mit demotischer Tintenaufschrift entpuppte (Reg. 617, Fig. 8). Die zweizeilige Aufschrift beinhaltet Titel und Namen des Besitzers bei Nennung seines Vaters und Großvaters: ${ }^{80}$

(1) jt-ntr $W 3 h$-jb-R ${ }^{c}$ s3 P3-dj-Jmn-[nsw]-t3.wj (?) (2) $s 3 W 3 h-j b-R^{e} p 3$ hm-ntr Jmn

(1) Der Gottesvater Ouaphres, Sohn des Petemestous (?), (2) Sohn des Ouaphres; der Amunpriester

Mit diesem Mumientäfelchen liegt nun der eindeutige Beweis vor, dass der aus der 26. Dynastie stammende Sarg Reg. 590 sekundär wiederverwendet wurde. Ob er dabei auch in seine Position innerhalb der Pfeilerhalle gebracht wurde, lässt sich nicht beweisen. Eine zeitliche Einordnung des zweiten Nutzers des Sarges kann hingegen erfolgen: Wie für die anderen Täfelchen aus dem Grab des Anch-Hor ist das 3. Jahrhundert v. Chr. anzunehmen. ${ }^{81}$ Somit können weder der als Besitzer, noch der als Großvater erwähnte Wahib-Re (= Ouaphres) mit dem Priester desselben Namens identisch sein, dessen intaktes Begräb-

\footnotetext{
79 Vgl. BietaK, Reiser-Haslauer 1982, 155.

${ }^{80}$ Für die Lesung der demotischen Aufschrift danke ich herzlich G. Vittmann.

81 S. Quaegebeur, in: Bietak, Reiser-Haslauer 1982, 266.

82 Der Besitzer des Täfelchens wäre dann Wah-ib-Re V; sein Großvater (Wah-ib-Re IV) könnte theoretisch mit
}

nis am Boden des Schachtes von Raum 10 entdeckt wurde. Die Zeitdifferenz von rund $80 \mathrm{Jah}-$ ren spricht eindeutig dagegen. Da die sterblichen Überreste des bekannten Wah-ib-Re I. in situ in seinen eigens angefertigten Särgen in Grabkammer 10.2 gefunden wurden, während hingegen das Mumientäfelchen zur Kennzeichnung einer Mumie in einem usurpierten, mit einem anderen Namen beschrifteten Sarg diente, kann eine Gleichsetzung zudem aus logischen Gründen ausgeschlossen werden. Damit liegt ein neuer Beleg für einen Gottesvater und Amunpriester namens Wah-ib-Re (Ouaphres) vor, der während des 3. Jahrhunderts v. Chr. in TT 414 bestattet wurde. ${ }^{82}$

Die verhältnismäßig hohe Anzahl ptolemäischer Mumientäfelchen aus TT 414 ist für den thebanischen Raum bislang singulär. ${ }^{83}$ Alle wurden in Raum 4, der Pfeilerhalle, gefunden und sie markieren wohl einen Zeithorizont, also eine Reihe von aufeinanderfolgende Bestattungen. Anders als in vorangehenden Epochen erfolgten Grablegungen nun nicht mehr ausschließlich in den unterirdischen Bestattungsräumen, sondern

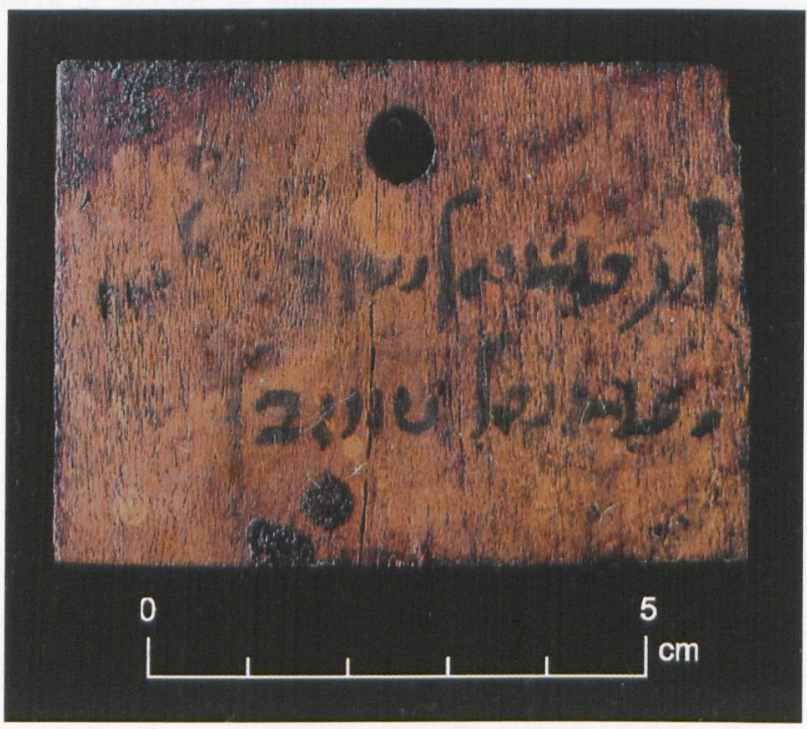

Fig. 8 Reg. 617 (09/04), Mumientäfelchen aus Reg. 590 nach der Reinigung (Foto J. Budka)

Wah-ib-Re III aus TT 414 identisch sein (s. BIETAK, REISER-Haslauer 1982, 271, G 44).

83 Allg. zu Mumientäfelchen s. QUAEgebeur 1978, 232259. Zu einem den Täfelchen aus TT 414 ähnlichen Stück (als spätptolemäisch datiert) s. GRAEFE 2003, 132, Kat. 85, Taf. 59,85. 
etliche Bestattungen wurden in den Räumen der unterirdischen Kultanlage eingebracht, wie neben der erwähnten Beschreibung von Lepsius auch die sekundäre Nutzung von Reg. 590 verdeutlicht. Die Vermutung liegt nahe, dass es sich bei den ptolemäischen Mumientäfelchen aus TT 414 um besondere Identifikationsmarken handelt, die dann zum Einsatz kamen, wenn die Grablegung in älteren, mit dem Namen des Vorbesitzers beschrifteten Särgen stattfand. Die am Toten befestigten Täfelchen konnten so unabhängig vom usurpierten Sarg den mumifizierten Körper identifizieren.

\section{ZUSAMMENFASSUNG}

Die Rekonstruktion von Bestattungsabläufen innerhalb eines Grabes sollte generell folgende Aspekte berücksichtigen: (1) die chronologische Abfolge der Grablegungen, (2) die sozialen Beziehungen der Bestatteten untereinander und (3) die Art der Bestattung. Letzteres schließt sowohl den Ort, als auch die Ausstattung und Sorte der Beigaben ein. ${ }^{84}$
Als vorläufiges Zwischenergebnis ist für TT 414 ein zwiespältiger Befund zur Nachnutzung und den Wiederbestattungen festzustellen, insbesondere in den beiden Bereichen Art und Ort der Bestattung (Tab. 1): (1) die Behandlung der Originalbestattungen ist ambivalent (Zerstörung, Recycling, profane Wiederverwendung und konzeptionelle Vorlagenfunktion); (2) es wurden sowohl originale Bestattungsanlagen wiederverwendet, als auch neue Kammern ausgemeißelt und andere Raumeinheiten zweckentfremdet.

Zur Art der Bestattung (1) ist also zum einen die Zerstörung und Dezimierung der Särge der Originalbestattungen in frühptolemäischer Zeit zu nennen, wie es insbesondere durch die profane Verbauung der Sarghölzer im „Lichthof" greifbar ist; zum anderen kam es im 3. Jahrhundert v. Chr. auch zur Wiederbenutzung anthropoider Särge in ihrer Funktion als Särge, wobei die älteren Stücke nicht beschädigt wurden. Darüber hinaus zeigen insbesondere frühptolemäische Särge „archaisierende“ Tendenzen und scheinen

\begin{tabular}{|c|c|c|c|c|c|}
\hline \multirow{2}{*}{ Phase } & \multirow{2}{*}{ Datierung } & \multirow{2}{*}{ Identität/Status } & \multicolumn{3}{|c|}{ Bestattungsmodus } \\
\hline & & & Art & Ort & Inventar \\
\hline $\begin{array}{l}\text { 1) Erstnutzung } \\
\text { und Bau der } \\
\text { Anlage }\end{array}$ & $\begin{array}{l}590-530 \\
\text { v. Chr. }\end{array}$ & $\begin{array}{l}\text { Familie des Anch-Hor } \\
\text { und weitere } \\
\text { Bedienstete der } \\
\text { Gottesgemahlin plus } \\
h m W s r-r\end{array}$ & $\begin{array}{l}\text { Primärbestattungen } \\
\text { in extra ange- } \\
\text { fertigten Räumen; } \\
\text { Nachbestattungen } \\
\text { der Familien- } \\
\text { angehörigen }\end{array}$ & $\begin{array}{l}\text { Rückwärtiger } \\
\text { Trakt: Schacht- } \\
\text { anlagen in R. } 7 \text {, } \\
8,9 \text { (auch } 10.1 \\
\text { im vorderen } \\
\text { Trakt?) }\end{array}$ & $\begin{array}{l}\text { Standardinventar mit } \\
\text { großen Qualitätsunter- } \\
\text { schieden (Särge, } \\
\text { Stelen, Uschebti, Kano- } \\
\text { pen, Schreine etc.) }\end{array}$ \\
\hline $\begin{array}{l}\text { 2) Wieder- } \\
\text { verwendung } \\
\text { plus Ausbau }\end{array}$ & $\begin{array}{l}380-300 \\
\text { v. Chr. }\end{array}$ & $\begin{array}{l}\text { Familie des Padi- } \\
\text { imen-neb-nesuttaui I } \\
\text { Amunpriester von } \\
\text { Karnak }\end{array}$ & $\begin{array}{l}\text { Sekundär- } \\
\text { bestattungen in } \\
\text { Grabkammer 7.1; } \\
\text { plus in neuen } \\
\text { Räumen (vgl. 10.2) }\end{array}$ & $\begin{array}{l}\text { v.a. R. } 7.1 \text {; } \\
\text { vorderer Trakt: } \\
\text { Ausbau Schacht } \\
\text { R. 10; ev. R. } 11\end{array}$ & $\begin{array}{l}\text { vgl. 26. Dyn. } \\
\text { („Archaismus“), krsw- } \\
\text { Ensemble; aber auch } \\
\text { neue Sargtypen + } \\
\text { Grabbeigaben }\end{array}$ \\
\hline $\begin{array}{l}\text { 3) Wiederver- } \\
\text { wendung und } \\
\text { Recycling; } \\
\text { Instandsetzung } \\
\text { des Lichthofs }\end{array}$ & $\begin{array}{l}300-150 \\
\text { v. Chr. }\end{array}$ & $\begin{array}{l}\text { Familie des Djed- } \\
\text { chensu-iuef-anch u.a. } \\
\text { Amunpriester von } \\
\text { Karnak }\end{array}$ & $\begin{array}{l}\text { Sekundär- } \\
\text { bestattungen in } \\
\text { vorhandenen } \\
\text { Räumen; Recycling } \\
\text { von Särgen }\end{array}$ & \multirow{2}{*}{$\begin{array}{l}\text { v. a. R. } 7.1 \text { und } \\
\text { R. 4: } \\
\text { Ausdehnung } \\
\text { der Bestattun- } \\
\text { gen im Bereich } \\
\text { der Kulträume }\end{array}$} & $\begin{array}{l}\text { „archaisierende“ } \\
\text { Formen plus Wieder- } \\
\text { verwendung von } \\
\text { Särgen; Mumientäfel- } \\
\text { chen als Erkennungs- } \\
\text { marken }\end{array}$ \\
\hline $\begin{array}{l}\text { 4) Wieder- } \\
\text { verwendung; } \\
\text { Ausweitung der } \\
\text { Bestattungszone }\end{array}$ & $\begin{array}{l}150 / 100-0 \\
\text { v. Chr. }\end{array}$ & $\begin{array}{l}\text { Familie des Osoroeris } \\
\text { und des Nesba-neb- } \\
\text { djedet u.a. } \\
\text { Amunpriester von } \\
\text { Karnak }\end{array}$ & $\begin{array}{l}\text { v.a. Okkupierung } \\
\text { der unterirdischen } \\
\text { Kulträume (auch R. } \\
\text { 2, ev. neu R. 3); tw. } \\
\text { Recycling }\end{array}$ & & $\begin{array}{l}\text { „archaisierende“ und } \\
\text { innovative Formen; } \\
\text { wohl Wiederverwen- } \\
\text { dung von Särgen; } \\
\text { aufrechte Aufstellung } \\
\text { der Särge }\end{array}$ \\
\hline
\end{tabular}

Tabelle 1 Skizze der Bestattungsabläufe in TT 414 (Saitisch bis Ptolemäisch)

$\overline{84}$ Vgl. BudKa 2010a, 364-367 (Fallbeispiel Grab VII im Asasif). 
saitische Vorlagen nachempfunden zu haben (vgl. Reg. 655, s.o.).

Die originalen Grabkammern im hinteren Bereich der Anlage, wohl vorzugsweise diejenige des Anch-Hor (Raum 7.1), wurden bereits seit dem 4. Jahrhundert v. Chr. bis in römische Zeit wiederverwendet - auffälligerweise von dem jeweiligen Familienoberhaupt, weshalb eine vorsätzliche Wertschätzung nahe liegt. Auch wenn die Reliefs im Lichthof zerstört, übermalt und unkenntlich gemacht wurden, so scheint man sich insbesondere der Bedeutung der Grabkammer 7.1 als ursprünglicher Bestattungsort des Anch-Hor bewusst gewesen zu sein. ${ }^{85}$

Zusätzlich ist für das 4. Jahrhundert v. Chr. aber auch, wie das Beispiel von Wah-ib-Re I. deutlich macht, ein Neuausbau von Schächten und Kammern festzustellen (Schacht $10 \mathrm{mit}$ Kammer 2). Künftige Untersuchungen sollen zeigen, ob sich hier schlicht ein Vielerlei der gelebten Bestattungspraxis widerspiegelt, ${ }^{86}$ oder aber die Lösung in der zeitlichen Differenz oder einem noch zu überprüfenden Statusunterschied der betreffenden Personen liegt. ${ }^{87}$ Möglich ist auch eine direkte Beeinflussung durch die Choachyten, beispielsweise Einschnitte aus finanziellen Überlegungen oder aufgrund von wechselnden Zuständigkeiten durch Verkauf oder Vererbung der Rechte an den Mumien oder dem Grab.

\section{Ausblick}

Zum Befund der wiederverwendeten saitischen Särge drängen sich einige Fragen auf: Wie ist es

${ }^{85}$ Das Grab des Harwa (TT 37) war beispielsweise bis ins 2. Jahrhundert v. Chr. in Papyri der Choachyten unter dem Namen seines Erbauers bekannt, s. Pestman 1993, 467-468, Anm. v und w. Ähnliches ist auch für TT 414 anzunehmen.

${ }^{86}$ Zum „Vielerlei der gelebten Wirklichkeit“ in einem anderen Kontext s. Seidlmayer 2003, 73. Vgl. auch BaINES, LACOVARA 2002, 5-36.

${ }^{87}$ Allerdings ist Wah-ib-Re I aus Schacht 10 wie der Besitzer des neuen Mumientäfelchens ein Gottesvater und Amunspriester (s. BietaK, Reiser-Haslauer 1982, 271, G 42), stammt also aus einem vergleichbaren beruflichem Umfeld und entsprechenden sozialen Verhältnissen.

88 Vgl. Pestman 1993, 454 für das normale Szenario, dass Abgaben dann aufhörten, wenn neue Familienoberhäupter bestattet wurden (Vater vs. Sohn); es gibt aber auch Fälle, wo lange gezahlt wurde, s. EBD., 450 für einen etwa zur Auswahl der Särge gekommen? Waren die Mumien der ursprünglichen Besitzer bereits beraubt und zerstört worden, oder erfolgte dies im Zuge der Wiederbenutzung? Waren die Särge von den Choachyten für Recycling freigegeben worden, da für die alten Mumien keine Abgaben mehr gezahlt wurden $?^{88}$ Die wohl wichtigsten Fragen kreisen um die Wertigkeit derartiger Wiederbestattungen - waren neue, eigens angefertigte Särge prestigeträchtiger als Altstücke? Anders formuliert, ist die Bestattung mit einer Identifikationsmarke in einem recycelten Sarg eine besonders Kosten sparende Variante? Oder assoziierte man mit einem „alten“ Sarg eines „Vorfahren" ebenfalls besondere Werte, möglicherweise weniger auf finanzieller, sondern auf emotionaler Ebene? ${ }^{89}$

Auffallend ist nämlich, dass keine Übermalungen durchgeführt wurden - weder stilistische Änderungen wurden vorgenommen, noch hat man die Namen der ursprünglichen Besitzer geändert. War dies alles eine Frage der Kosten und der ökonomischen Nutzung? ${ }^{90}$ Oder eine individuelle Frage des Geschmacks? Ein Resultat des Faktors Zufall? Eine Mischung verschiedener Faktoren? Oder doch - man denke an den bewussten Einsatz der Mumientäfelchen - Teil eines komplexen Regelwerks? Hinzu kommt, dass in TT 414 einige Personen desselben Namens aus unterschiedlichen Epochen belegt sind ${ }^{91}$ - ist auch dies ein zufälliger Befund, oder liegt hier eine systematische Steuerung durch die Nekropolenverwaltung bzw. die Choachyten oder auch ein Wunsch der zu Bestattenden vor?

Beleg, dass für zwei 153 v. Chr. eingebrachte Mumien noch 40 Jahre später die Ausgaben beglichen wurden.

${ }^{89}$ Vgl. etwa die sicher bewusste und wohl kaum auf finanziellen Gründen basierende Wiederbenutzung des Sarges von Thutmosis I. durch Pinudjem I., s. TAYLOR 1992, 191

90 Vgl. das gängige Recycling diverser Grabbeigaben während der 21. Dynastie (v.a. Särge und Uschebtis), s. TAYLOR 1992, 190-200; JANSEN-WINKELN 1995, 72 und 75.

91 Vgl. z.B. Reiser-Haslauer, in: Bietak, Reiser-Haslauer 1982, 269-270 für mehrere ptolemäische Personen namens $J r t-H r-r w$, also Namensvetter zum saitischen Besitzer von Reg. 590. Für die Beliebigkeit des Befundes spricht allerdings, dass Reg. 590 nicht von einer dieser Personen wiederverwendet wurde, sondern von einem Wah-ib-Re. 
Die zahlreichen Texte, die aus ptolemäischer Zeit überliefert sind und von einem streng geregelten Bestattungswesen Zeugnis ablegen ${ }^{92}$ könnten dafür sprechen, dass der uns heute so fragmentarisch und ambivalent wirkende Befund in TT 414 nichtsdestotrotz die Resultante eines übergeordneten Regelwerkes ist. Nicht auszuschließen sind aber individuelle „Fehler" oder regelwidrige Verhaltensmuster, die den Befund zusätzlich geprägt haben. Gerade in ptolemäischer Zeit muss man verstärkt von einem wirtschaftlichen Interesse an Mumien und Bestattungen ausgehen - möglicherweise sind die unterschiedlichen Behandlungen auch durch verschiedene Höhen an Zuwendungen an die Choachyten zu erklären. ${ }^{93}$ Insgesamt sind mit der massiven Nachnutzung der saitischen Tempelgräber im Asasif ganz offensichtlich noch zahlreiche offene Fragen verbunden.

\section{Bibliographie}

ARP, J.

2009 Zur Methodik der Analyse von Gräbern in der Ägyptologie, unpublizierte Dissertation, Ludwig-Maximilians-Universität München.

Assmann, J.

1968 Arbeiten im Grab des B3s3, in: D. ARnOLd, J. SetTGAST, Vierter Vorbericht über die vom Deutschen Archäologischen Institut Kairo im Asasif unternommenen Arbeiten, MDAIK 23, 20-25.

2001 Tod und Jenseits im alten Ägypten, München.

2005 Die Lebenden und die Toten, 16-36, in: J. Assmann, F. Maciejewski, A. Michaels (Hgg.), Der Abschied von den Toten. Trauerrituale im Kulturvergleich, Göttingen.

Aston, D.A.

2003 The Theban West Bank from the Twenty-fifth Dynasty to the Ptolemaic Period, 138-166, in: N. Strudwick, J. H. TAYlor (Hgg.), The Theban Necropolis, Past, Present and Future, London.

2009 Burial Assemblages of Dynasty 21-25. Chronology Typology-Developments, CChEM 21, Wien.
Anhand des Fallbeispiels TT 414 soll künftig auch untersucht werden, ob die uns als Besonderheiten erscheinenden Befunde deshalb hervorstechend, weil in der Anlage vielfältige Belege für die heterogene Wiederbenutzung über mehrere Jahrhunderte vorliegen und wir zudem zeitgleiche Texte als Informationsquellen nützen können. Ist es allein die bessere Quellenlage, der wir das ambivalente Bild verdanken? Oder kann vielleicht doch von einem immanenten Charakterzug der altägyptischen Kultur gesprochen werden, nämlich dass sich auch bei einem stringenten Regelwerk Lücken auftun? Denn materielle Hinterlassenschaften gehen letztendlich auf konkrete Personen und deren spezifischen Entscheidungen zurück, die bei allen möglichen Vorgaben doch als Individuen agierten und menschliche Stärken und Schwächen ebenso wie Ideenreichtum einbringen konnten.

Baines, J., Lacovara, P.

2002 Burial and the dead in ancient Egyptian society: respect, formalism, neglect, Journal of Social Archaeology 2, 2002, 5-36.

BERNBECK, R.

1997 Theorien in der Archäologie, Tübingen und Basel.

Bietak, M., Reiser-Haslauer, E.

1978 Das Grab des Anch-Hor, Obersthofmeister der Gottesgemahlin Nitokris, Band I. Mit einem Beitrag von Erhart Graefe und Relief- und Fundzeichnungen von Heinz Satzinger, UZK 4, Wien.

1982 Das Grab des Anch-Hor, Obersthofmeister der Gottesgemahlin Nitokris, Band II. Mit Beiträgen von Joachim Boessneck, Angela von den Driesch, Jan Quaegebeur, Helga Liese-Kleiber und Helmut Schlichtherle und Relief- und Fundzeichnungen von Heinz Satzinger, UZK 5, Wien.

Bierbrier, M. L.

1987 Hieroglyphic Texts from Egyptian Stelae etc., Part 11, BMP, London.

93 Darüber hinaus muss man einkalkulieren, dass Zuwendungen einer Familie zum Erliegen kamen; vgl. PEsTMAN 1993, 454 und passim. 
BROVARKSI, E.

1978 Canopic Jars, CAA, Museum of Fine Arts, Boston, Fasc. 1, Mainz/Rhein 1978.

BUDKA, J.

2006 Die Spätzeit in Theben-West: Das Asasif. Bestattungsbrauchtum und Friedhofsstruktur anhand der Ergebnisse der österreichischen Ausgrabungen in den Jahren 19691977, unpublizierte Dissertation, Universität Wien.

2007 Tomb VII in the Asasif: Its owners, date and implications, 241-250, in: J. Goyon, C. CARdin (Hgg.), Proceedings of the Ninth International Congress for Egyptologists in Grenoble, OLA 150, vol. I, Leuven.

2008a Neues zu den Nutzungsphasen des Monumentalgrabes von Anch-Hor, Obersthofmeister der Gottesgemahlin Nitokris (TT 414), $\ddot{A} \mathcal{E} L$ 18, 61-85.

2008b Wiedersehen mit Anch-Hor: Neue Arbeiten in der österreichischen Konzession des Asasifs (ThebenWest), Sokar 16, 74-79.

2009a Neues zum Grab des Anch-Hor (TT 414, Asasif): Der „Lichthof", Sokar 18, 80-87.

2009b Ankh-Hor Revisited: Study, Documentation and Publication of Forgotten Finds from the Asasif/ Thebes, 23-31, in: J. Popielska, J. Iwaszczuk (Hgg.), Fifth Central European Conference of Egyptologist. Egypt 2009: Perspectives of Research, Pultusk 2009, Acta Archaeologica Pultuskiensia, Pułtusk.

2010a Bestattungsbrauchtum und Friedhofsstruktur im Asasif. Eine Untersuchung der spätzeitlichen Befunde anhand der Ergebnisse der österreichischen Ausgrabungen in den Jahren 1969-1977, UZK 34, Wien.

2010b Wiederbestattungen im Grab des Anch-Hor (TT 414): Neufunde der Kampagne 2009, Sokar 20, 86-91.

im Druck Fundmaterial aus Gräbern: Möglichkeiten und Grenzen der archäologischen Interpretation und ihre didaktische Vermittlung, in: A. VerbovseK, B. BaCKes, C. Jones (Hgg.), Ägyptologie und Kulturwissenschaft, Akten des Symposiums München 2008 [Heidelberg, 2010].

BURKARD, G.

1986 Grabung im Asasif 1963-1970, Band III, Die Papyrusfunde. Nach Vorarbeiten von Dido Bidoli $(\dagger)$, AV 22, Mainz am Rhein.

CoOney, K. M.

2007 The Cost of Death. The social and economic value of Ancient Egyptian funerary art in the Ramesside Period, EU 22, Leiden.

DoDson, A., IKRAM, S.

2008 The Tomb in Ancient Egypt: Royal and Private Sepulchres from the Early Dynastic Period to the Romans, Kairo.

EIGNER, D.

1984 Die monumentalen Grabbauten der Spätzeit in der Thebanischen Nekropole, UZK 6, Wien.

\section{FITZENREITER, M.}

1994 Zum Ahnenkult in Ägypten, GM 143, 51-72.

2008 Jenseits im Diesseits - Die Konstruktion des Ortes der Toten im pharaonischen Ägypten, 75-106, in: C. Kümmel, B. Schweizer, U. VeIt (Hgg.), Körperinszenierung - Objektsammlung - Monumentalisierung: Totenritual und Grabkult in frühen Gesellschaften. Archäologische Quellen in kulturwissenschaftlicher Perspektive, Tübinger Archäologische Taschenbücher 6, Münster u.a.

Gestermann, L.

2006 Das spätzeitliche Schachtgrab als memphitischer Grabtyp, 195-206, in: G. Moers et al. (Hgg.), jn.t dr.w. Festschrift für Friedrich Junge, Göttingen.

GNIRS, A. M.

2003 Der Tod des Selbst. Die Wandlung der Jenseitsvorstellungen in der Ramessidenzeit, 173-199, in: H. Guksch, E. Hofmann, M. Bommas (Hgg.), Grab und Totenkult im Alten Ägypten, München.

Graefe, E.

2003 Das Grab des Padihorresnet, Obervermögensverwalter der Gottesgemahlin des Amun (Thebanisches Grab Nr. 196), mit Beiträgen von Jan Quaegebeur (†), Peter Dils und Diethelm Eigner, Monumenta Aegyptiaca IX, Tournhout.

GRAJETZKI, W.

2003 Burial Customs in Ancient Egypt: Life in Death for Rich and Poor, London.

GuksCH, H.

1995 Über den Umgang mit Gräbern, 13-24, in: Thebanische Beamtennekropolen, Neue Perspektiven archäologischer Forschung, Internationales Symposion Heidelberg 9.-13.6. 1993, SAGA 12, Heidelberg.

Hurcombe, L.

2007 Archaeological Artefacts as Material Culture, London und New York 2007.

JÁNOSI, P.

2005 Giza in der 4. Dynastie, Die Baugeschichte und Belegung einer Nekropole des Alten Reiches, Band I: Die Mastabas der Kernfriedhöfe und die Felsgräber, UZK 24, Wien.

JANSEN-WINKELN, K.

1995 Die Plünderung der Königsgräber des Neuen Reiches, ZÄS 122, 62-78.

KAMPP, F.

1996 Die Thebanische Nekropole. Zum Wandel des Grabgedankens von der XVIII. bis zur XX. Dynastie, Theben 13, Mainz am Rhein.

KAMPP-SEYFRIED, F.

2003 Es lebt Re-Harachte, der im Lichtland jubelt. Ein „Glaubensbekenntnis“ ohne Worte aus der Nachamarnazeit, 118-127, in: H. GuKsch, E. Hofmann, M. Bommas (Hgg.), Grab und Totenkult im Alten Ägypten, München. 


\section{KITCHEN, K.A.}

1979 Memphite tomb-chapels in the New Kingdom and later, 272-284, in: M. GÖRG, K.J. SEYFrIEd (Hgg.), Festschrift Elmar Edel, 12. März 1979, ÄAT 1, Bamberg.

KuHLMANN, K.-P.

1973. Eine Beschreibung der Grabdekoration mit der Aufforderung zu kopieren und zum Hinterlassen von Besucherinschriften aus saitischer Zeit, MDAIK 29, 205-213.

de Meulenaere, $\mathrm{H}$.

1989 Notes de prosopographie thébaine, Quatrième série, CdÉ 64, 55-73.

MunRo, P.

1973 Die spätägyptischen Totenstelen, ÄF 25, Glückstadt.

NEUREITER, S.

1995 Eine neue Interpretation des Archaismus, SAK 21, 219-254.

Pestman, P. W.

1993 The Archive of the Theban Choachytes (Second Century B.C.), Studia Demotica II, Leuven.

Polz, D.

1987 Excavation and recording of a Theban tomb: some remarks on recording methods, in: J. AssMANN et al. (Hgg.), Problems and Priorities in Egyptian Archaeology, Studies in Egyptology, London, 119-140.

1990 Bemerkungen zur Grabbenutzung in der thebanischen Nekropole, MDAIK 46, 301-336.

QuAEgEBeur, J.

1978 Mummy labels: an Orientation, 232-259, in: E. Boswinkel, P.W. Pestman (Hgg.), Texts grecs, démotiques et bilingues, Papyrologica Lugduno-Batava 19, Leiden.

1982 VIII. Demotic Inscriptions on Wood from the Tomb of 'Anch-Hor, 259-266, in: M. BIETAK, E. REISER-HASLAUER 1982.

QUIRKE, S.

1993 Owners of Funerary Papyri in the British Museum, British Museum Occasional Paper 92, London.

RichaRds, J. E.

2005 Society and Death in Ancient Egypt: Mortuary Landscapes of the Middle Kingdom, Cambridge.

ROCCATI, A.

1995 Rückgriff auf ältere Traditionen im Dekorationsprogramm von TT 27, 81-84, in: Thebanische Beamtennekropolen, Neue Perspektiven archäologischer Forschung, Internationales Symposion Heidelberg 9.-13.6. 1993, SAGA 12, Heidelberg.

SATZINGER, H,

1979 Theben, 95-114, in: Funde aus Ägypten. Österreichische Ausgrabungen seit 1961, Katalog KHM, Wien.

\section{SCHIFFER, M. B.}

1972 Archaeological context and systemic context, American Antiquity 37, 156-165.

1987 Formation Processes of the Archaeological Record, Albuquerque.

SCHreIBer, G.

2008 The Mortuary Monument of Djehutymes II, Finds from the New Kingdom to the Twenty-Sixth Dynasty, Studia Aegyptiaca Series Maior II, Budapest.

SEIDLMAYER, S. J.

2003 Vom Sterben der kleinen Leute. Tod und Bestattung in der sozialen Grundschicht am Ende des Alten Reiches, 60-74, in: H. Guksch, E. Hofmann, M. Bommas (Hgg.), Grab und Totenkult im Alten Ägypten, München.

2009 Prestigegüter im Kontext der Breitenkultur im Ägypten des 3. und 2. Jahrtausends v. Chr., 309334, in: B. Hildebrandt, C. Veit (Hgg.), Der Wert der Dinge - Güter im Prestigediskurs, „Formen von Prestige in Kulturen des Altertums“, Graduiertenkolleg der DFG an der Ludwig-Maximilians-Universität München, Münchner Studien zur Alten Welt 6, München.

SEILER, A.

2005 Tradition $\mathcal{E}^{2}$ Wandel. Die Keramik als Spiegel der Kulturentwicklung in der Zweiten Zwischenzeit, SDAIK 32, Mainz am Rhein.

SEYFRIED, K. J.

1990 Das Grab des Amonmose (TT 373), Theben 4, Mainz am Rhein.

1995 Das Grab des Djehutiemhab (TT 194), Theben 7, Mainz am Rhein.

SPENCER, A. J.

1982 First and Second Owners of a Memphite Tomb Chapel, JEA 68, 20-26.

STAMmers, M.

2009 The Elite Late Period Egyptian Tombs of Memphis, BAR International Series 1903, Oxford.

STRUDWICK, N.

1996 The Tombs of Amenhotep, Khnummose, and Amenmose at Thebes (nos. 294, 253 and 254), Oxford.

2003 Some aspects of the archaeology of the Theban necropolis in the Ptolemaic and Roman periods, 167-188, in: N. Strudwick, J.H. TAYLor (Hgg.), The Theban Necropolis, Past, Present and Future, London.

TAYLOR, J. H.

1989 Egyptian Coffins, Shire Egyptology 11, Aylesbury.

1992 Aspects of the History of the Valley of the Kings in the Third Intermediate Period, 186-206, in: C.N. ReEves (Hg.), After Tutankhamun. Research and excavation in the Royal Necropolis at Thebes, Studies in Egyptology, London/New York. 
2003 Theban coffins from the Twenty-second to the Twenty-sixth Dynasty: dating and synthesis of development, 95-121, in: N. Strudwick, J.H. TAYLOR (Hgg.), The Theban Necropolis, Past, Present and Future, London.

2010 Changes in the Afterlife, 220-240, in: W. WENDRICH (Hg.), Egyptian Archaeology, Malden, Oxford \& Chichester.
Vleeming, S. P.

1995 The Office of a Choachyte in the Theban Area, 241-255, in: S.P. VleEming (Hg.), Hundred-Gated Thebes. Acts of a Colloquium on Thebes and the Theban Area in the Graeco-Roman Period (= P. L. Bat. 27), Leiden/New York/Köln.

WINLOCK, H. E.

1932 The Tomb of Queen Meryet-Amūn at Thebes, Publications of The Metropolitan Museum of Art, Egyptian Expedition VI, New York. 